\title{
Soret and Dufour Effects on Heat and Mass Transfer of Boundary Layer Flow over Porous Wedge with Thermal Radiation: Bivariate Spectral Relaxation Method
}

\author{
Felix Ilesanmi Alao, Chika Uchechukwu Boneze, Adeyemi Isaiah Fagbade
}

Department of Mathematical Sciences, Federal University of Technology, Akure, Nigeria

Email address:

sanmialao@gmail.com (F. I. Alao), bonezechika@gmail.com (C. U. Boneze), yemi2favours@yahoo.co.uk (A. I. Fagbade)

To cite this article:

Felix Ilesanmi Alao, Chika Uchechukwu Boneze, Adeyemi Isaiah Fagbade. Soret and Dufour Effects on Heat and Mass Transfer of Boundary Layer Flow over Porous Wedge with Thermal Radiation: Bivariate Spectral Relaxation Method. American Journal of Chemical Engineering. Vol. 7, No. 1, 2019, pp. 7-21. doi: 10.11648/j.ajche.20190701.12

Received: January 9, 2019; Accepted: March 2, 2019; Published: March 25, 2019

\begin{abstract}
Boundary layer flow has great importance in engineering applications such as oil bed recovery, filtration, thermal insulations, heat exchangers, geothermal analysis and so on. This paper investigated the effects of heat radiation, soret and dufour in the presence of suction or injection on a boundary layer flow over a porous wedge. The governing equations with the boundary conditions are non-dimensionalized by introducing some non-dimensional variables. The flow model is described in terms of a highly coupled and nonlinear system of partial differential equations as the method of solution seeks to decouple the original system to form a sequence of equations corresponding to the momentum, energy and concentration equations that is solved in a computationally efficient manner. The resulting equations are solved using a numerical technique called Bivariate Spectral Relaxation Method (BSRM). Numerical calculations are carried out for different values of dimensionless parameters and the analysis of the physical parameters of engineering applications are investigated. Effects of these major parameters on transport behaviors are investigated and typical results are illustrated to reveal the effect of pertinent parameter on the velocity, temperature, concentration profiles of the flow. The effects on the local skin friction, local nusselt and Sherwood number are also presented in the tables.
\end{abstract}

Keywords: Chemical Reaction, Heat Radiation, Porous Wedge, Soret and Dufour, Bivariate Spectral Relaxation Method (BSRM)

\section{Introduction}

Thermal radiations on flow and heat processes are of major importance in the design of many advanced energy conversion systems operating at high temperature. Thermal radiation within these systems is usually the result of emission by hot walls and the working fluid. The present trend in the field of chemical reaction analysis is to give a mathematical model for a system to predict the reactor performance. A large amount of research work has been reported in this field. In particular, the study of heat and mass transfer with chemical reaction is of considerable importance in chemical and hydro-metallurgical industries. The fluid properties are assumed to be constant and a first-order homogeneous chemical reaction is assumed to take place in the flow. Chemical reaction can be classified as heterogeneous or homogeneous. A homogeneous chemical reaction is one that occurs uniformly throughout a given phase. The species generation in a homogeneous reaction is analogous to internal source of heat generation. Suction or injection on the boundary layer played significant role in the field of aerodynamics and space sciences.

Shojaefard [1] used suction/injection to control fluid flow on the surface of subsonic aircraft. Braslow [2] stated that by controlling the flow such as in fuel consumption might be decreased by $30 \%$, a considerable reduction in pollutant emission is achieved and operating costs of commercial airplanes are reduced by at least $8 \%$. Sudhagar et al [3] explored the association of mixed convection flows with convective boundary conditions on heat and mass transfers of nanofluid in an isothermal vertical wedge implanted in a nonDarcy porous medium. The model used for the nanofluid 
consolidates the impact of Brownian motion, thermophoresis, and convective boundary conditions. Many interests have been shown in the study of flow of heat and mass transfer with suction or injection because of its extensive engineering applications.

The heat and mass transfer simultaneously affecting each other that will cause the cross-diffusion effect. The heat transfer caused by concentration gradient is called the diffusion-thermo or dufour effect. On the other hand, mass transfer caused by temperature gradients is called soret or thermal diffusion effect. Thus soret effect is referred to species differentiation developing in an initial homogenous mixture submitted to a thermal gradient and the dufour effect referred to the heat flux produced by a concentration gradient. The soret effect, for instance has been utilized for isotope separation, and also in the mixture between gases with very light molecular weight $\left(H_{2}, H_{e}\right)$ and of medium molecular weight $\left(N_{2}\right.$, Air $)$. The importance of soret and dufour effects is in fluids with very light and medium molecular weight. In heat and mass transfer problems, the variation of density with temperature and concentration give rise to a combined buoyancy force under natural convection. Soret and dufour effects are also critical in various porous flow regimes occurring in chemical and geophysical systems.

A common area of interest in the field of aerodynamics is the analysis of thermal boundary layer problems for twodimensional steady and incompressible laminar flow passing a wedge. Simultaneous heat and mass transfer from different geometrics embedded in porous media has many engineering and geophysical applications such as geothermal reservoirs, drying of porous solids, thermal insulation, enhanced oil recovery, packed-bed catalytic reactors, cooling of nuclear reactors, and underground energy transport.

Moorthy [4] analyzed soret and dufour effects on natural convection heat and mass transfer flow past a horizontal surface in a porous medium with variable viscosity. It was evident that for gases, the Nusselt number decreases and the Sherwood number increases as it is opposite for liquids. Shivaiah and Rao [5] dealt with the effects of heat and mass transfer on two-dimensional steady MHD free convection flow along a vertical porous plate embedded in porous medium in presence of thermal radiation, heat generation, viscous dissipation and chemical reaction under the influence of dufour and soret effects. These similar equations were then solved numerically by using the shooting method along with fourth order Runge - Kutta integration scheme. It was also assumed that all the fluid properties were constant except that of the influence of the density variation with temperature and concentration in the body force term (Boussinesq's approximation). The soret term exemplifies the temperature gradient effects on the variation of concentration, hence the decrease.

Omowaye et al. [6] investigated a magnetohydrodynamic boundary layer problem posed when the viscosity of the fluid varied inversely with temperature. It revealed the influence of temperature dependent fluid viscosity parameter on dufour, soret and magnetic parameter. The non-linear momentum, energy and species boundary layer equations were transformed into ordinary differential equations using suitable similarity variables. The transformed boundary layer equations were solved using HAM. Rashidi [7] studied the MHD mixed convective heat transfer for an incompressible, laminar, and electrically conducting viscoelastic fluid flow past a permeable wedge with thermal radiation via a semianalytical/numerical method, called Homotopy Analysis Method (HAM). The boundary layer governing partial differential equations were transformed into highly nonlinear coupled ordinary differential equations consisting of the momentum and energy equations using similarity solution. Muhaimin [8] investigated the effect of thermophoresis particle deposition and temperature dependent viscosity on unsteady non-Darcy mixed convective heat and mass transfer of a viscous and incompressible fluid past a porous wedge in the presence of chemical reaction. The wall of the wedge is embedded in a uniform non-Darcian porous medium in order to allow for possible fluid wall suction or injection.

Lavanya and Ratnam [9] focused on the effects of soret, dufour and thermal radiation on unsteady MHD free convection flow past an infinite vertical porous plate in the presence of chemical reaction has been analyzed. The dimensionless governing equations were solved numerically by a Finite Element Method. The velocity, temperature and concentration profiles were presented for different parameters. Kandasamy et al [10] studied the effects of chemical reaction, heat and mass transfer on boundary layer flow over a porous wedge with heat radiation in the presence of suction or injection. The study showed an approximate numerical solution for the steady laminar boundary layer flow over a wall of the wedge in the presences of species concentration and mass diffusion which were obtained by solving the governing equations using numerical techniques. The flow field was influenced by the chemical reaction, buoyancy ratio between species and thermal diffusion and suction/injection at the wall surface.

Stagnation flows are found in many applications such as flows over the tips of rockets, aircrafts, submarines and oil ships. Transport processes in porous media play a significant role in various applications, such as geothermal engineering, thermal insulation, energy conservation, petroleum industries and solid matrix heat exchangers. Wubshet and Ayele [11] discovered the problem of two-dimensional steady laminar MHD boundary layer flow past a wedge with heat and mass transfer of nanofluid embedded in porous media with viscous dissipation, Brownian motion, and thermophoresis effect. The transmuted model was shown to be controlled by a number of thermophysical parameters, viz. the pressure gradient, magnetic, permeability, Prandtl number, Lewis number, Brownian motion, thermophoresis, and Eckert number.

Motsa et al [12] illustrated the implementation of Bivariate Spectral Relaxation Method, where it analyzed fluid viscosity unsteady boundary layer flow over a vertical surface due to impulsive motion. Suitable similarity variables were used to transform the governing equations to yield a highly non- 
linear and coupled system of partial differential equations which was decoupled using Gauss-Siedel relaxation technique and the implementation of spectral collocation in both space and time variable.

From the literature survey, the influence of thermal radiation, soret and dufour on the boundary layer flow over a porous wedge in the presence of chemical reaction have not been investigated so far. Hence, we result to study using a BSRM approach. The transformed governing equations are solved using the bivariate spectral relaxation method.

\section{Mathematical Formulation}

A steady two-dimensional laminar convective boundary layer flow over a porous wedge in the presence of soret, dufour and thermal radiation is considered. The $x$-axis is taken parallel to the wedge and $y$-axis is taken normal to the wedge. The fluid is assumed to be Newtonian e. $g$, Air, Water $\left(\mathrm{H}_{2} \mathrm{O}\right)$.

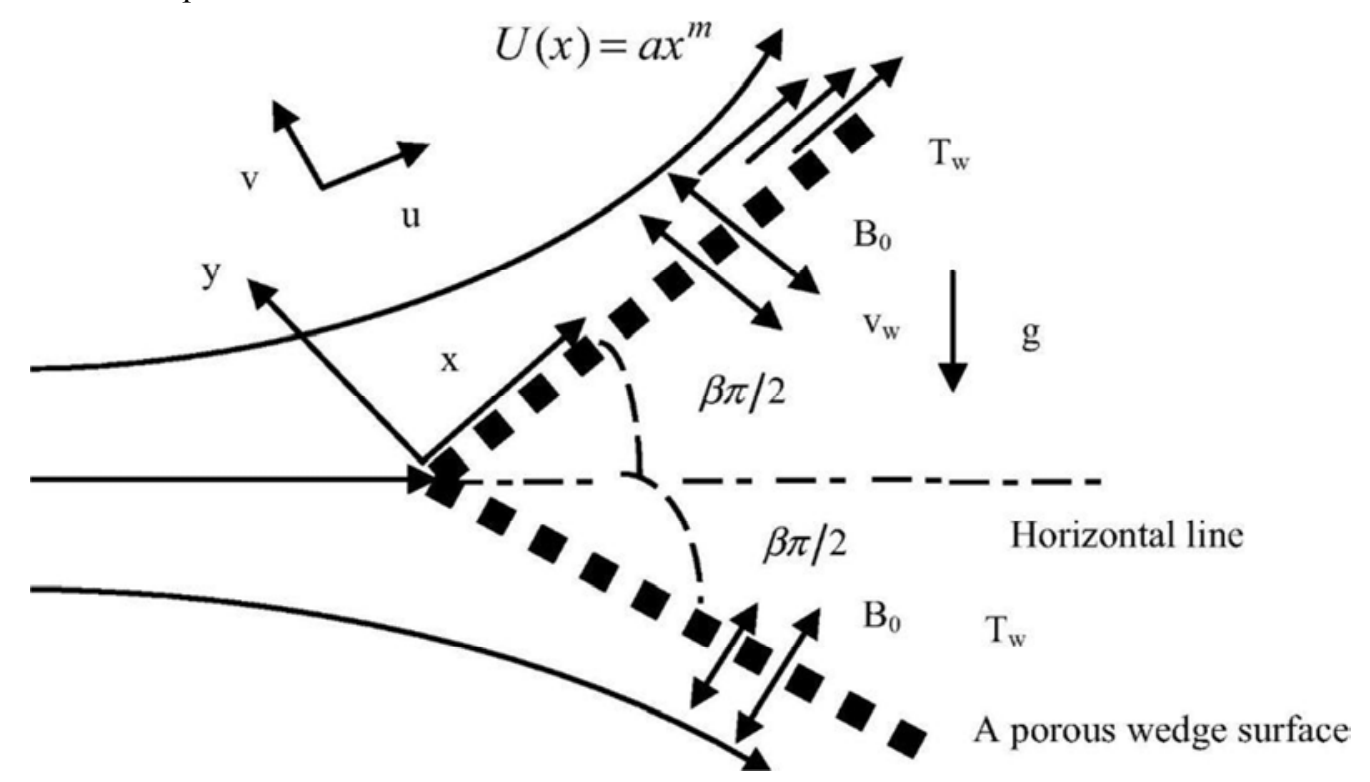

Figure 1. Flow analysis along the wall of the porous wedge.

The density variation and the effects of buoyancy are taken into consideration in the momentum equation (Boussinesq's approximation [13]). The chemical reactions are taken place in the flow and a constant suction or injection will be imposed at the surface of the wedge. The effects of sorets and dufour in the effects of chemical reaction, heat and mass transfer on boundary layer flow over a porous wedge with heat radiation in the presence of suction or injection. Now, the governing boundary layer equations of momentum, energy and diffusion for the flow under Boussinesq's approximation are:

$$
\frac{\partial u}{\partial x}+\frac{\partial v}{\partial y}=0
$$

Subjected to the boundary conditions;

$$
\begin{aligned}
& u=0, v=v_{0}, C=C_{w}, T=T_{w} \text { aty }=0, \text {, } \\
& \left.u=U(x), C=C_{\infty}, T=T_{\infty} \text { asy } \rightarrow \infty .\right\}
\end{aligned}
$$

where all the variables and parameters are defined under nomenclature.

The terms in the governing equations are defined below:

$\vartheta \frac{\partial^{2} \mathrm{u}}{\partial \mathrm{y}^{2}}$ is viscous term, $\mathrm{U}_{0} \frac{\mathrm{dU}_{0}}{\mathrm{dx}}$ is pressure term, $\left[g \beta_{T}(T-\right.$ $\left.\left.T_{\infty}\right)+g \beta_{C}\left(C-C_{\infty}\right)\right] \sin \frac{\Omega}{2}$ is buoyancy forces along the wedge,
$\frac{\vartheta}{\mathrm{K}}\left(\mathrm{u}-\mathrm{U}_{0}\right)$ is porosity term along the wedge, $\propto \frac{\partial^{2} \mathrm{~T}}{\partial \mathrm{y}^{2}}$ is thermal diffusivity, $\frac{1}{\rho \mathrm{C}_{\mathrm{p}}} \frac{\partial \mathrm{q}_{\mathrm{r}}}{\partial \mathrm{y}}$ is radiative heat flux, $\frac{\mathrm{D}_{\mathrm{m}}}{\mathrm{C}_{\mathrm{s}}} \frac{\mathrm{k}_{\mathrm{T}}}{\mathrm{c}_{\mathrm{p}}} \frac{\partial^{2} \mathrm{C}}{\partial \mathrm{y}^{2}}$ is dufour terms, $\frac{Q_{0}}{\rho C_{p}}\left(T-T_{\infty}\right)$ is heat absorbed or generated, $\mathrm{D}_{\mathrm{m}} \frac{\partial^{2} \mathrm{C}}{\partial \mathrm{y}^{2}}$ is mass diffusivity, $\mathrm{K}_{\mathrm{r}}\left(\mathrm{C}-\mathrm{C}_{\infty}\right)$ is concentration term, $\frac{\mathrm{D}_{\mathrm{m}} \mathrm{K}_{\mathrm{T}}}{\mathrm{T}_{\mathrm{m}}} \frac{\partial^{2} \mathrm{~T}}{\partial \mathrm{y}^{2}}$ is soret term.

Using Roseland approximation, the radiative heat flux is given by: 


$$
q_{r}=-\frac{4 \sigma}{3 \mu} \frac{\partial T^{4}}{\partial y}
$$

where $\sigma$ and $\mu$ are the Stefan-Boltzmann constant and the mean absorption coefficient respectively. By using Roseland approximation, the present study is limited to optically thick fluids. If temperature differences within the flow are sufficiently small, then we are going to linearize equation (6) by expanding $T^{4}$ in the Taylor series about $T_{\infty}$ and neglecting higher order term.

Neglecting the higher order terms, it has been shown that: $T^{4} \approx 4 T T_{\infty}^{3}-3 T_{\infty}^{4}$. Therefore, equation (6) becomes

$$
\frac{\partial q_{r}}{\partial y}=-\frac{16 \sigma}{3 \mu} T_{\infty}^{3}\left(\frac{\partial^{2} T}{\partial y^{2}}\right)
$$

Substitute (7) into the energy equation (3), which becomes equation (8)

$$
u \frac{\partial T}{\partial x}+v \frac{\partial T}{\partial y}=\propto \frac{\partial^{2} T}{\partial y^{2}}+\frac{16 \sigma}{3 \rho C_{p} \mu} T_{\infty}^{3} \frac{\partial^{2} T}{\partial y^{2}}+\frac{D_{m}}{C_{s}} \frac{k_{T}}{c_{p}} \frac{\partial^{2} C}{\partial y^{2}}+\frac{Q_{0}}{\rho C_{p}}\left(T-T_{\infty}\right)
$$

Following the lines of Kafoussias et al., [14], the following change of variables are introduced

$$
\psi(x, y)=\sqrt{\frac{2 U_{0} v x}{1+m}} f(x, \eta) ; \theta=\frac{T-T_{\infty}}{T_{w}-T_{\infty}} ; \eta(x, y)=y \sqrt{\frac{(1+m) U_{0}}{2 v x}} ; \phi=\frac{\mathrm{C}-\mathrm{C}_{\infty}}{\mathrm{C}_{\mathrm{w}}-\mathrm{C}_{\infty}}
$$

Under this consideration, the potential flow velocity can be written as

$$
U_{0}(x)=A x^{m}, \beta_{1}=\frac{2 m}{1+m}
$$

Where $A, m$ are constants. $\beta_{1}$ is the Hartree pressure gradient parameter that corresponds to $\beta_{1}=\frac{\Omega}{\pi}$ for a total angle $\Omega$ of the wedge.

The continuity equation (1) is satisfied by the stream function $\psi(x, y)$ defined by

$$
u=\frac{\partial \psi}{\partial y} \mathrm{and} v=-\frac{\partial \psi}{\partial x}
$$

The equations (2) to (4) become the set of coupled non-linear partial differential equations;

$$
\begin{gathered}
\frac{\partial^{3} f}{\partial \eta^{3}}+\left(f+\beta_{2} x \frac{\partial f}{\partial x}\right)\left(\frac{\partial^{2} f}{\partial \eta^{2}}\right)-\beta_{1} x \frac{\partial f}{\partial \eta}\left(\frac{\partial^{2} f}{\partial x \partial \eta}\right)+\beta_{2}\left(1-\left(\frac{\partial f}{\partial \eta}\right)^{2}\right)+\beta_{2} \operatorname{Cr}(\theta+N \phi) \operatorname{Sin}\left(\frac{\Omega}{2}\right)+\beta_{2} \lambda\left(\frac{\partial f}{\partial \eta}-1\right)=0 \\
(1+R) \frac{\partial^{2} \theta}{\partial \eta^{2}}+\operatorname{RPr} f \frac{\partial \theta}{\partial \eta}-\operatorname{RPr} \beta_{2} \theta \frac{\partial f}{\partial \eta}-\operatorname{RPr} \beta_{2} x\left(\frac{\partial f}{\partial \eta} \frac{\partial \theta}{\partial x}-\frac{\partial f}{\partial x} \frac{\partial \theta}{\partial \eta}\right)+R \operatorname{Pr} \beta_{2} \delta \theta+R D u \frac{\partial^{2} \phi}{\partial \eta^{2}}=0 \\
\frac{\partial^{2} \phi}{\partial \eta^{2}}+S c f \frac{\partial \phi}{\partial \eta}-S c \gamma \beta_{2} x \phi-S c \beta_{2} \phi\left(\frac{\partial f}{\partial \eta}\right)-S c \beta_{2} x\left(\frac{\partial f}{\partial \eta} \frac{\partial \phi}{\partial x}-\frac{\partial f}{\partial x} \frac{\partial \phi}{\partial \eta}\right)+S r \frac{\partial^{2} \theta}{\partial \eta^{2}}=0
\end{gathered}
$$

The corresponding boundary conditions are:

$$
\begin{gathered}
\frac{\partial f}{\partial \eta}=0, f(\xi, 0)=S 1, \theta=1, \phi=1 \\
\frac{\partial f}{\partial \eta} \rightarrow 1, \theta \rightarrow 0, \phi \rightarrow 0
\end{gathered}
$$

We now introduce these parameters;

$$
\begin{gathered}
\beta_{1}=\frac{2 m}{1+m} ; \beta_{2}(\text { constant })=\frac{2}{1+m} ; \operatorname{Pr}=\frac{\vartheta}{\propto} ; \delta=\left(\frac{Q_{0} x}{\rho C_{p} U_{o}}\right) \\
G r=\left(\frac{g \beta\left(T-T_{\infty}\right)}{\left(A x^{m}\right)^{2}}\right) ; N=\frac{g \beta^{*}\left(C-C_{\infty}\right)}{g \beta\left(T-T_{\infty}\right)} ; \lambda=\frac{\beta}{k}\left(\frac{1}{A x^{m}}\right) ; \gamma=\frac{k_{r}}{A x^{m}} \\
R=\frac{3 \rho C_{p} \propto \mu}{16 \sigma T_{\infty}^{3}} ; D u=\frac{D_{m}}{\propto C_{s}} \frac{k_{T}}{C_{p}}\left(\frac{C_{w}-C_{\infty}}{T_{w}-T_{\infty}}\right) ; S c=\frac{\vartheta}{D_{m}} ; S r=\frac{D_{m} K_{T}}{\vartheta T_{m}}\left(\frac{T_{w}-T_{w}}{C_{w}-C_{\infty}}\right)
\end{gathered}
$$

Where $\mathrm{v}_{0}$ is the velocity of suction if $\mathrm{v}_{0}<0$ and injection if $\mathrm{v}_{0}>0$ also $\mathrm{S} 1$ is the suction parameter if $S 1>0$ and injection if $S 1<0$. Let $\xi=k x^{\frac{1-m}{2}}$ [14] be the dimensionless distance along the porous wedge surface $(\xi>0)$. In this system of equations $f(\xi, \eta), \theta(\xi, \eta)$ and $\phi(\xi, \eta)$ are the dimensionless stream function, dimensionless temperature and dimensionless concentration respectively. Both the wall temperature and concentration are assumed to have powerlaw variation forms as shown by the following equations:

$$
\left.\begin{array}{l}
T_{w}=T_{\infty}+C_{1} x^{n}, \\
C_{w}=C_{\infty}+C_{2} x^{n} .
\end{array}\right\}
$$


where $C_{1}$ and $C_{2}$ are constants and $n$ is the power of index of the wall temperature $(T)$ and concentration $(C)$.

Equations (12) to (16) now result into the following:

$$
\begin{gathered}
\frac{\partial^{3} f}{\partial \eta^{3}}+\left(f+\beta_{2} \xi \frac{\partial f}{\partial \xi}\right)\left(\frac{\partial^{2} f}{\partial \eta^{2}}\right)-\beta_{1} \xi \frac{\partial f}{\partial \eta}\left(\frac{\partial^{2} f}{\partial \xi \partial \eta}\right)+\beta_{2}\left(1-\left(\frac{\partial f}{\partial \eta}\right)^{2}\right)+\beta_{2} \operatorname{Gr}(\theta+N \phi) \operatorname{Sin}\left(\frac{\Omega}{2}\right)+\beta_{2} \lambda\left(\frac{\partial f}{\partial \eta}-1\right)=0 \\
(1+R) \frac{\partial^{2} \theta}{\partial \eta^{2}}+R \operatorname{Pr} f \frac{\partial \theta}{\partial \eta}-\operatorname{RPr} \beta_{2} \theta \frac{\partial f}{\partial \eta}-\operatorname{RPr} \beta_{2} \xi\left(\frac{\partial f}{\partial \eta} \frac{\partial \theta}{\partial \xi}-\frac{\partial f}{\partial \xi} \frac{\partial \theta}{\partial \eta}\right)+R \operatorname{Pr} \beta_{2} \delta \theta+R D u \frac{\partial^{2} \phi}{\partial \eta^{2}}=0 \\
\frac{\partial^{2} \phi}{\partial \eta^{2}}+S c f \frac{\partial \phi}{\partial \eta}-S c \gamma \beta_{2} \xi^{2} \phi-S c \beta_{2} \phi\left(\frac{\partial f}{\partial \eta}\right)-S c \beta_{2} \xi\left(\frac{\partial f}{\partial \eta} \frac{\partial \phi}{\partial \xi}-\frac{\partial f}{\partial \xi} \frac{\partial \phi}{\partial \eta}\right)+S r \frac{\partial^{2} \theta}{\partial \eta^{2}}=0
\end{gathered}
$$

The corresponding boundary conditions are:

$$
f(\xi, 0)=\left(\frac{2}{1+m}\right) \xi^{\frac{1-m}{2}}\left(S-\xi^{\frac{1+m}{2}} \frac{\partial f}{\partial \xi} \xi(\xi, 0)\right)=S 1
$$

$S 1>0$ corresponds to suction and $S 1<0$ corresponds to injection.

$$
\begin{gathered}
\frac{\partial f}{\partial \eta}=0, f(\xi, 0)=S_{1}, \theta=1, \phi=1 \\
\frac{\partial f}{\partial \eta} \rightarrow 1, \theta \rightarrow 0, \phi \rightarrow 0
\end{gathered}
$$

Equations (18) to (20) with the boundary conditions can be regarded as a system of partial differential equations for the functions $f, \theta$ and $\phi$ with $\xi$ as a parameter for given pertinent parameters. In practical engineering application, major important physical parameters of practical values are the local skin friction coefficient, Nusselt number and Sherwood number for this boundary layer flow. There are defined as follows:

$\tau_{w}=\mu\left(\frac{\partial u}{\partial y}\right)_{y=0}$ and in dimensionless form, we obtain $C_{f}=$ $\frac{\tau_{w}}{\rho U_{0}^{2}}$

Therefore, the local friction is: $C_{f}=\frac{\mu}{\rho U_{0} x}\left(\sqrt{\frac{(1+m)}{2}}\right) R e_{x}^{1 / 2} f^{\prime \prime}(\xi, 0)$

The Nusselt number is given by: $N u=-\frac{x}{\left(T_{w}-T_{\infty}\right)}\left(\frac{\partial T}{\partial y}\right)_{y=0}$

$$
\begin{gathered}
\mathrm{g}_{\mathrm{r}+1}^{\prime \prime}+a_{0, \mathrm{r}}(\xi, \eta)+a_{2, \mathrm{r}}(\xi, \eta) g_{r+1}^{\prime}-\beta_{2} \lambda+a_{1, \mathrm{r}}(\xi, \eta) \mathrm{g}_{\mathrm{r}+1}^{\prime}-\beta_{1} g_{r}^{2}=a_{3, \mathrm{r}}(\xi, \eta) \frac{\partial \mathrm{g}_{\mathrm{r}+1}}{\partial \xi}+a_{4, \mathrm{r}}(\xi, \eta) \mathrm{g}_{\mathrm{r}+1}^{\prime}\left(\frac{\partial \mathrm{f}_{\mathrm{r}+1}}{\partial \xi}\right) \\
f_{r+1}^{\prime}=g_{r+1} \\
b_{0, \mathrm{r}}(\xi, \eta) \theta_{r+1}^{\prime \prime}+b_{1, \mathrm{r}}(\xi, \eta) \theta_{r+1}+b_{2, \mathrm{r}}(\xi, \eta)+b_{3, \mathrm{r}}(\xi, \eta) \theta_{r+1}^{\prime}+b_{4, \mathrm{r}}(\xi, \eta) \theta_{r+1}=b_{5, \mathrm{r}}(\xi, \eta) \frac{\partial \theta_{r+1}}{\partial \xi}\left(-b_{6, \mathrm{r}}(\xi, \eta) \theta_{r+1}^{\prime} \frac{\partial \mathrm{f}_{\mathrm{r}+1}}{\partial \xi}\right) \\
\phi_{r+1}^{\prime \prime}+c_{0, \mathrm{r}}(\xi, \eta) \phi_{r+1}+c_{1, \mathrm{r}}(\xi, \eta)+c_{2, \mathrm{r}}(\xi, \eta) \phi_{r+1}^{\prime}+c_{3, \mathrm{r}}(\xi, \eta) \phi_{r+1}=c_{4, \mathrm{r}}(\xi, \eta) \frac{\partial \phi_{r+1}}{\partial \xi}+c_{5, \mathrm{r}}(\xi, \eta) \phi_{r+1}^{\prime} \frac{\partial \mathrm{f}_{\mathrm{r}+1}}{\partial \xi}
\end{gathered}
$$

Subject to the boundary conditions:

$$
\left.\begin{array}{c}
\mathrm{f}_{\mathrm{r}+1}(\xi, 0)=S 1 ; \mathrm{g}_{\mathrm{r}+1}(\xi, 0)=0 ; \theta_{\mathrm{r}+1}(\xi, 0)=1 ; \phi_{\mathrm{r}+1}(\xi, 0)=1 \\
\mathrm{~g}_{\mathrm{r}+1}(\xi, \infty)=1 ; \theta_{\mathrm{r}+1}(\xi, \infty)=0 ; \phi_{\mathrm{r}+1}(\xi, 0)=0
\end{array}\right\}
$$

Where the coefficients are defined as

$$
\begin{gathered}
a_{0, \mathrm{r}}(\xi, \eta)=\beta_{2}+\beta_{2} \operatorname{Gr}\left(\theta_{r}+N \phi_{r}\right) \sin \left(\frac{\Omega}{2}\right)-\beta_{2} \lambda ; a_{1, \mathrm{r}}(\xi, \eta)=\beta_{2} \lambda ; a_{2, \mathrm{r}}(\xi, \eta)=\mathrm{f}_{\mathrm{r}} ; \\
a_{3, \mathrm{r}}(\xi, \eta)=\beta_{2} \xi \mathrm{g}_{\mathrm{r}} ; a_{4, \mathrm{r}}(\xi, \eta)=-\beta_{2} \xi ; a_{5, \mathrm{r}}(\xi, \eta)=-\beta_{1} g_{r}^{2} ;
\end{gathered}
$$




$$
\begin{gathered}
b_{0, \mathrm{r}}(\xi, \eta)=(1+\mathrm{R}) ; b_{1, \mathrm{r}}(\xi, \eta)=\operatorname{Pr} R \beta_{2} \delta ; b_{2, \mathrm{r}}(\xi, \eta)=R D u \phi_{r}^{\prime \prime} ; b_{3, \mathrm{r}}(\xi, \eta)=\operatorname{Pr} R \mathrm{f}_{\mathrm{r}+1} ; \\
b_{4, \mathrm{r}}(\xi, \eta)=-\operatorname{Pr} R \beta_{2} \theta_{r+1} ; b_{5, \mathrm{r}}(\xi, \eta)=\operatorname{Rr} \beta_{2} \xi g_{r+1} ; b_{6, \mathrm{r}}(\xi, \eta)=-R \operatorname{Pr} \beta_{2} \xi ; \\
c_{0, \mathrm{r}}(\xi, \eta)=-\xi^{2} \operatorname{Sc\gamma } \beta_{2} ; c_{1, \mathrm{r}}(\xi, \eta)=\operatorname{Sr} \theta_{r+1}^{\prime \prime} ; c_{2, \mathrm{r}}(\xi, \eta)=S c \mathrm{f}_{\mathrm{r}+1} ; \\
c_{3, \mathrm{r}}(\xi, \eta)=-S c \beta_{2} g_{r+1} ; c_{4, \mathrm{r}}(\xi, \eta)=\xi \beta_{2} S c g_{r+1} ; c_{5, \mathrm{r}}(\xi, \eta)=-\xi \beta_{2} S c
\end{gathered}
$$

Motsa et al [15] used spectral collocation method to discretise in space $\eta$ and finite differences in time domain $\xi$. But this paper discretised in space $\eta$ and time $\xi$ domains. The chebyshev collocation method requires the domain to be transformed to $[-1,1] \times[-1,1]$. With linear transformation of $\eta \in[-1,1]$ and $\xi \in[-1,1]$ to $\tau \in[-1,1]$ and $\zeta \in[-1,1]$, respectively. The spatial and time domains are discretized using Chebyshev-Gauss-Lobatto points defined as:

$$
\tau_{i}=\cos \left(\frac{\pi i}{N_{x}}\right), \zeta_{i}=\cos \left(\frac{\pi i}{N_{t}}\right), i=0,1, \ldots, N_{x} ; j=0,1, \ldots, N_{t}
$$

Each equation in the decoupled linear system of partial differential equations (18) - (20) is solved independently. In solving equation (18) it is assumed to be approximated by a bivariate lagrange interpolation polynomial of the form:

$$
g(\xi, \eta) \approx \sum_{m=0}^{N_{x}} \sum_{j=0}^{N_{t}} \mathrm{~g}\left(\tau_{m}, \zeta_{j}\right) \mathrm{L}_{\mathrm{m}}\left(\tau_{k}\right) \mathrm{L}_{\mathrm{j}}(\zeta)
$$

It interpolates $g(\xi, \eta)$ at the collocation points.

$$
g(\xi, \eta) \approx \sum_{m=0}^{N_{x}} \sum_{j=0}^{N_{t}} \mathrm{~g}\left(\tau_{m}, \zeta_{j}\right) \mathrm{L}_{\mathrm{m}}\left(\tau_{k}\right) \mathrm{L}_{\mathrm{j}}(\zeta)
$$

Function

$$
\mathrm{L}_{\mathrm{m}}(\tau)=\prod_{\substack{m=0 \\
m \neq k}}^{N_{x}} \frac{\tau-\tau_{k}}{\tau_{m}-\tau_{k}}, \mathrm{~L}_{\mathrm{m}}\left(\tau_{k}\right)=\delta_{\mathrm{m}, \mathrm{k}}=\left\{\begin{array}{c}
0 \text { if } m \neq k \\
1 \text { ifm }=\mathrm{k}
\end{array}\right.
$$

Function $L_{j}(\zeta)$ is defined in the same way.

Following (Canuto et al., [16],Trefethen [17]) , we define the derivatives of $g(\xi, \eta)$ with respect to $\eta$ and $\xi$ at the collocation points $\tau_{k}$ and $\zeta_{i}$ as follows:

$$
\begin{gathered}
\left.\frac{\partial g}{\partial \eta}\right|_{\left(\tau_{k}, \zeta_{i}\right)}=\frac{2}{\eta_{\infty}} \sum_{m=0}^{N_{x}} \sum_{j=0}^{N_{t}} \mathrm{~g}\left(\tau_{m}, \zeta_{j}\right) \frac{\mathrm{dL}\left(\tau_{k}\right)}{\mathrm{d} \tau} \mathrm{L}_{\mathrm{j}}\left(\zeta_{i}\right)=\mathrm{DG}_{\mathrm{i}} \\
\left.\frac{\partial^{2} g}{\partial \eta^{2}}\right|_{\left(\tau_{k}, \zeta_{i}\right)}=D^{2} \mathrm{G}_{\mathrm{i}} \\
\left.\frac{\partial g}{\partial \xi}\right|_{\left(\tau_{k}, \zeta_{i}\right)}=2 \sum_{m=0}^{N_{x}} \sum_{j=0}^{N_{t}} \mathrm{~g}\left(\tau_{m}, \zeta_{j}\right) \frac{\mathrm{d} \mathrm{L}_{\mathrm{j}}\left(\zeta_{i}\right)}{\mathrm{d} \zeta} \mathrm{L}_{\mathrm{m}}\left(\tau_{k}\right)=2 \sum_{j=0}^{N_{t}} \mathrm{~d}_{\mathrm{i}, \mathrm{j}} \mathrm{G}_{\mathrm{j}}
\end{gathered}
$$

Where $\mathrm{d}_{\mathrm{i}, \mathrm{j}}\left(\mathrm{i}, \mathrm{j}=0,1,2, \ldots, N_{t}\right)$ are entries of the standard Chebyshev differentiation matrix $d=\left[d_{i, j}\right]$ of size $\left(N_{t}+1\right) \times$ $\left(N_{t}+1\right)$ see for an example (Canutoetal., [16],Trefethen [17]), $D=\frac{2}{\eta_{e}}\left[D_{r, s}\right]\left(r, s=0,1, \ldots, N_{x}\right)$ with $\left[D_{r, s}\right]$ being a $\left(N_{x}+1\right) \times\left(N_{x}+1\right)$ Chebyshev derivative matrix and the vector $\mathrm{G}_{\mathrm{i}}$ is defined as

$$
\mathrm{G}_{\mathrm{i}}=\left[\mathrm{g}_{\mathrm{i}}\left(\tau_{0}\right), \mathrm{g}_{\mathrm{i}}\left(\tau_{1}\right), \ldots, \mathrm{g}_{\mathrm{i}}\left(\tau_{N_{x}}\right)\right]^{\mathrm{T}}
$$

Accordingly, applying the collocation method, we arrive at;

$$
A_{i} \mathrm{G}_{\mathrm{r}+1, \mathrm{i}}+a_{0, \mathrm{r}}\left(\xi_{\mathrm{i}}\right)+a_{5, \mathrm{r}}-2 a_{3, \mathrm{r}}\left(\xi_{\mathrm{i}}\right) \sum_{\mathrm{j}=0}^{\mathrm{Nt}} \mathrm{d}_{\mathrm{i}, \mathrm{j}} \mathrm{G}_{\mathrm{r}+1, \mathrm{j}}-2 a_{4, \mathrm{r}}\left(\xi_{\mathrm{i}}\right) D \mathrm{G}_{\mathrm{r}+1, \mathrm{i}} \sum_{\mathrm{j}=0}^{\mathrm{Nt}} \mathrm{d}_{\mathrm{i}, \mathrm{j}} \mathrm{f}_{\mathrm{r}+1}=0
$$

Subject to boundary conditions

$$
\mathrm{g}_{\mathrm{r}+1, \mathrm{i}}\left(\tau N_{x}\right)=0, \mathrm{~g}_{\mathrm{r}+1, \mathrm{i}}\left(\tau_{0}\right)=1
$$

Where $A_{i}=\mathrm{D}^{2}+a_{2, \mathrm{r}}\left(\xi_{\mathrm{i}}\right) \mathrm{D}+a_{1, \mathrm{r}}\left(\xi_{\mathrm{i}}\right)$

$a_{\mathrm{m}, \mathrm{r}}\left(\xi_{\mathrm{i}}\right)(\mathrm{m}=0,1)$ is the diagonal matrix of the vector $\left[a_{\mathrm{m}, \mathrm{r}}\left(\tau_{0}\right), a_{\mathrm{m}, \mathrm{r}}\left(\tau_{1}\right), \ldots, a_{\mathrm{m}, \mathrm{r}}\left(\tau N_{x}\right)\right]^{\mathrm{T}}$ and $a_{2, \mathrm{r}}\left(\xi_{\mathrm{i}}\right)=\left[a_{2, \mathrm{r}}\left(\tau_{0}\right), a_{2, \mathrm{r}}\left(\tau_{1}\right), \ldots, a_{2, \mathrm{r}}\left(\tau N_{x}\right)\right]^{\mathrm{T}}$.

Expanding equation (37) and impose boundary conditions for $i=0,1, \ldots, \mathrm{N}_{\mathrm{t}}$ gives the following matrix equation:

$$
\left[\begin{array}{ccc}
A_{0,0} & \cdots & A_{0, \mathrm{~N}_{\mathrm{t}}} \\
\vdots & \ddots & \vdots \\
A_{\mathrm{N}_{\mathrm{t}}, 0} & \cdots & A_{\mathrm{N}_{\mathrm{t}}, \mathrm{N}_{\mathrm{t}}}
\end{array}\right]\left[\begin{array}{c}
G_{\mathrm{r}+1,0} \\
G_{\mathrm{r}+1,1} \\
\vdots \\
G_{\mathrm{r}+1, \mathrm{~N}_{\mathrm{t}}}
\end{array}\right]=\left[\begin{array}{c}
R_{1,0} \\
R_{1,1} \\
\vdots \\
R_{1, \mathrm{~N}_{\mathrm{t}}}
\end{array}\right]
$$

Where

$$
\begin{gathered}
A_{i, i}=A_{i}-2 a_{3, \mathrm{r}}\left(\xi_{\mathrm{i}}\right) \mathrm{d}_{\mathrm{i}, \mathrm{j}}-2 a_{4, \mathrm{r}}\left(\xi_{\mathrm{i}}\right) D . \mathrm{I} . \mathrm{d}_{\mathrm{i}, \mathrm{j}} \mathrm{I} \text {, wheni }=0,1, \ldots, \mathrm{N}_{\mathrm{t}-1} \\
A_{i, j}=-2 a_{3, \mathrm{r}}\left(\xi_{\mathrm{i}}\right) \mathrm{d}_{\mathrm{i}, \mathrm{j}} \mathrm{I}-2 a_{4, \mathrm{r}}\left(\xi_{\mathrm{i}}\right) D . \mathrm{I}_{\mathrm{i}, \mathrm{j}} \mathrm{I} \text {, when } \mathrm{i} \neq \mathrm{j}
\end{gathered}
$$




$$
R_{1, i}=-a_{0, \mathrm{r}}\left(\xi_{\mathrm{i}}\right)-a_{5, \mathrm{r}}\left(\xi_{\mathrm{i}}\right)
$$

Where I is an $\left(N_{x}+1\right) \times\left(N_{x}+1\right)$ identity matrix

Similarly, applying the bivariate collocation on equations (26) and (27) gives

$$
\begin{aligned}
& B_{\mathrm{i}} \theta_{\mathrm{r}+1, \mathrm{i}}+b_{2, \mathrm{r}}-2 b_{5, \mathrm{r}} \sum_{\mathrm{j}=0}^{\mathrm{Nt}} \mathrm{d}_{\mathrm{i}, \mathrm{j}} \theta_{\mathrm{r}+1, \mathrm{j}}-2 b_{6, \mathrm{r}} D \theta_{\mathrm{r}+1, \mathrm{i}} \sum_{\mathrm{j}=0}^{\mathrm{Nt}} \mathrm{d}_{\mathrm{i}, \mathrm{j}} \mathrm{f}_{\mathrm{r}+1, \mathrm{j}}=0 \\
& C_{\mathrm{i}} \phi_{\mathrm{r}+1, \mathrm{i}}+c_{1, \mathrm{r}}-2 c_{4, \mathrm{r}} \sum_{\mathrm{j}=0}^{\mathrm{Nt}} \mathrm{d}_{\mathrm{i}, \mathrm{j}} \phi_{\mathrm{r}+1, \mathrm{j}}-2 c_{5, \mathrm{r}} D \phi_{\mathrm{r}+1, \mathrm{i}} \sum_{\mathrm{j}=0}^{\mathrm{Nt}} \mathrm{d}_{\mathrm{i}, \mathrm{j}} \mathrm{f}_{\mathrm{r}+1, \mathrm{j}}=0
\end{aligned}
$$

Subject to the boundary conditions

$$
\theta_{\mathrm{r}+1, \mathrm{i}}\left(\tau N_{x}\right)=0, \theta_{\mathrm{r}+1, \mathrm{i}}\left(\tau_{0}\right)=1, \phi_{\mathrm{r}+1, \mathrm{i}}\left(\tau N_{x}\right)=0, \phi_{\mathrm{r}+1, \mathrm{i}}\left(\tau_{0}\right)=1
$$

Where $B_{i}=b_{0, r}\left(\xi_{\mathrm{i}}\right) \mathrm{D}^{2}+b_{1, \mathrm{r}}\left(\xi_{\mathrm{i}}\right) \mathrm{D}+b_{3, \mathrm{r}}\left(\xi_{\mathrm{i}}\right) \mathrm{D}+b_{4, \mathrm{r}}\left(\xi_{\mathrm{i}}\right)$

$$
C_{i}=\mathrm{D}^{2}+c_{0, \mathrm{r}}\left(\xi_{\mathrm{i}}\right) \mathrm{D}+c_{2, \mathrm{r}}\left(\xi_{\mathrm{i}}\right) \mathrm{D}+c_{3, \mathrm{r}}\left(\xi_{\mathrm{i}}\right)
$$

$b_{\mathrm{m}, \mathrm{r}}\left(\xi_{\mathrm{i}}\right), c_{\mathrm{m}, \mathrm{r}}\left(\xi_{\mathrm{i}}\right)(\mathrm{m}=0,1)$ are the diagonal matrix of the vector $\left[b_{\mathrm{m}, \mathrm{r}}\left(\tau_{0}\right), b_{\mathrm{m}, \mathrm{r}}\left(\tau_{1}\right), \ldots, b_{\mathrm{m}, \mathrm{r}}\left(\tau N_{x}\right)\right]^{\mathrm{T}}$ and $\left[c_{\mathrm{m}, \mathrm{r}}\left(\tau_{0}\right), c_{\mathrm{m}, \mathrm{r}}\left(\tau_{1}\right), \ldots, c_{\mathrm{m}, \mathrm{r}}\left(\tau N_{x}\right)\right]^{\mathrm{T}}, b_{2, \mathrm{r}}\left(\xi_{\mathrm{i}}\right)=\left[b_{2, \mathrm{r}}\left(\tau_{0}\right), b_{2, \mathrm{r}}\left(\tau_{1}\right), \ldots, b_{2, \mathrm{r}}\left(\tau N_{x}\right)\right]^{\mathrm{T}}$ and $\left[c_{2, \mathrm{r}}\left(\tau_{0}\right), c_{2, \mathrm{r}}\left(\tau_{1}\right), \ldots, c_{2, \mathrm{r}}\left(\tau N_{x}\right)\right]^{\mathrm{T}}$.

Expanding equation (43) and (44) and impose boundary conditions for $i=0,1, \ldots, \mathrm{N}_{\mathrm{t}}$ gives the following matrix equation:

$$
\begin{gathered}
{\left[\begin{array}{ccc}
B_{0,0} & \cdots & B_{0, \mathrm{~N}_{\mathrm{t}}} \\
\vdots & \ddots & \vdots \\
B_{\mathrm{N}_{\mathrm{t}}, 0} & \cdots & B_{\mathrm{N}_{\mathrm{t}}, \mathrm{N}_{\mathrm{t}}}
\end{array}\right]\left[\begin{array}{c} 
\\
\theta_{\mathrm{r}+1,0} \\
\theta_{\mathrm{r}+1,1} \\
\theta_{\mathrm{r}+1, \mathrm{~N}_{\mathrm{t}}}
\end{array}\right]=\left[\begin{array}{c}
R_{2,0} \\
R_{2,1} \\
: \\
R_{2, \mathrm{~N}_{\mathrm{t}}}
\end{array}\right]} \\
{\left[\begin{array}{ccc}
C_{0,0} & \cdots & C_{0, \mathrm{~N}_{\mathrm{t}}} \\
\vdots & \ddots & \vdots \\
C_{\mathrm{N}_{\mathrm{t}}, 0} & \cdots & C_{\mathrm{N}_{\mathrm{t}}, \mathrm{N}_{\mathrm{t}}}
\end{array}\right]\left[\begin{array}{c} 
\\
\phi_{\mathrm{r}+1,0} \\
\phi_{\mathrm{r}+1,1} \\
\vdots \\
\phi_{\mathrm{r}+1, \mathrm{~N}_{\mathrm{t}}}
\end{array}\right]=\left[\begin{array}{c}
R_{3,0} \\
R_{3,1} \\
\vdots \\
R_{3, \mathrm{~N}_{\mathrm{t}}}
\end{array}\right]}
\end{gathered}
$$

Where

$$
\begin{gathered}
B_{i, i}=B_{\mathrm{i}}-2 b_{5, \mathrm{r}} \mathrm{d}_{\mathrm{i}, \mathrm{j}} \mathrm{I}-2 b_{6, \mathrm{r}} D \cdot \mathrm{I} \cdot \mathrm{d}_{\mathrm{i}, \mathrm{I}} \mathrm{I} \mathrm{i}=0,1, \ldots, \mathrm{N}_{\mathrm{t}} \\
B_{i, j}=-2 b_{5, \mathrm{r}} \mathrm{d}_{\mathrm{i}, \mathrm{j}} \mathrm{I}-2 b_{6, \mathrm{r}} \text { D. I. } \mathrm{d}_{\mathrm{i}, \mathrm{i}} \mathrm{I} \text { when } \mathrm{i} \neq \mathrm{j} \\
R_{2, i}=-\left(b_{2, \mathrm{r}}\right), R_{3, i}=-\left(c_{1, \mathrm{r}}\right) \\
R_{2, i}=-\left(b_{2, \mathrm{r}}\right), R_{3, i}=-\left(c_{1, \mathrm{r}}\right) \\
C_{i, i}=C_{i}-2 c_{4, \mathrm{r}} \mathrm{d}_{\mathrm{i}, \mathrm{j}} \mathrm{I}-2 c_{5, \mathrm{r}} \text { D. I. } \mathrm{d}_{\mathrm{i}, \mathrm{j}} \mathrm{I}, \mathrm{i}=0,1, \ldots, \mathrm{N}_{\mathrm{t}-1} \\
C_{i, j}=-2 c_{4, \mathrm{r}} \mathrm{d}_{\mathrm{i}, \mathrm{j}} \mathrm{I}-2 c_{5, \mathrm{r}} D . \mathrm{I} \cdot \mathrm{d}_{\mathrm{i}, \mathrm{j}} \mathrm{I} \text {, when } \mathrm{i} \neq \mathrm{j}
\end{gathered}
$$

The approximate solutions for $g(\xi, \eta), \theta(\xi, \eta)$ and $\phi(\xi, \eta)$ are obtained by iteratively solving the matrix equations, (39), (46) and (47) in turn for $r=$ $0,1,2, \ldots$ Simple exponent functions that satisfy the boundary conditions (22) and (23) can be used as initial approximations to start the iterative process at $r=0$. In this work, these functions were used as initial approximations:

$f_{0}(\xi, \eta)=-1+\eta+e^{-\eta}+S 1, \theta_{0}(\xi, \eta)=e^{-\eta} \operatorname{and} \phi_{0}(\xi, \eta)=e^{-\eta}$

The equations are further coded using symbolic mathematical software from MatLab to solve the flow equations numerically with Bivariate Spectral Relaxation Method.

\section{Discussion of Results}

In order to get physical insight into the problem, the numerical calculations for the distribution of the velocity, temperature, concentration, across the boundary layer for various values of the parameter have been carried out using bivariate spectral relaxation method on the governing equations and the corresponding boundary conditions. The effects of the main controlling parameters as they appear in the governing equations are discussed in the current section. In the present study, the following default parametric values are adopted $S 1=1 ; \gamma=0.5 ; \mathrm{Pr}=0.71 ; \mathrm{N}=2 ; \mathrm{Gr}=0.5 ; \mathrm{R}=$ $0.5 ; \delta=0.5 ; \mathrm{Sc}=0.62 ; \mathrm{Sr}=0.5 ; \Omega=(\mathrm{pi} / 6) ; \xi=0.5 ; \lambda=0.5$; $\mathrm{Du}=0.4$. All graphs therefore correspond to these unless specifically indicated on the appropriate graph.

The influence of the chemical reactiony on velocity profiles has been illustrated in Figure 2. It is observed that, while all other participating parameters are held constant and $\gamma$ is increased, it is seen that the velocity decreases with increase in chemical reaction. Figure 3, shows the influence of $\gamma$ on the temperature profile of the flow where it increases as the chemical reaction $(\gamma)$ increases. The Figure 4 portrays the 
effects of chemical reaction on the concentration of the flow profile. It is shown that the concentration of the fluid decreases with increase in the chemical reaction. This shows that diffusion rate can be tremendously altered by chemical reaction.

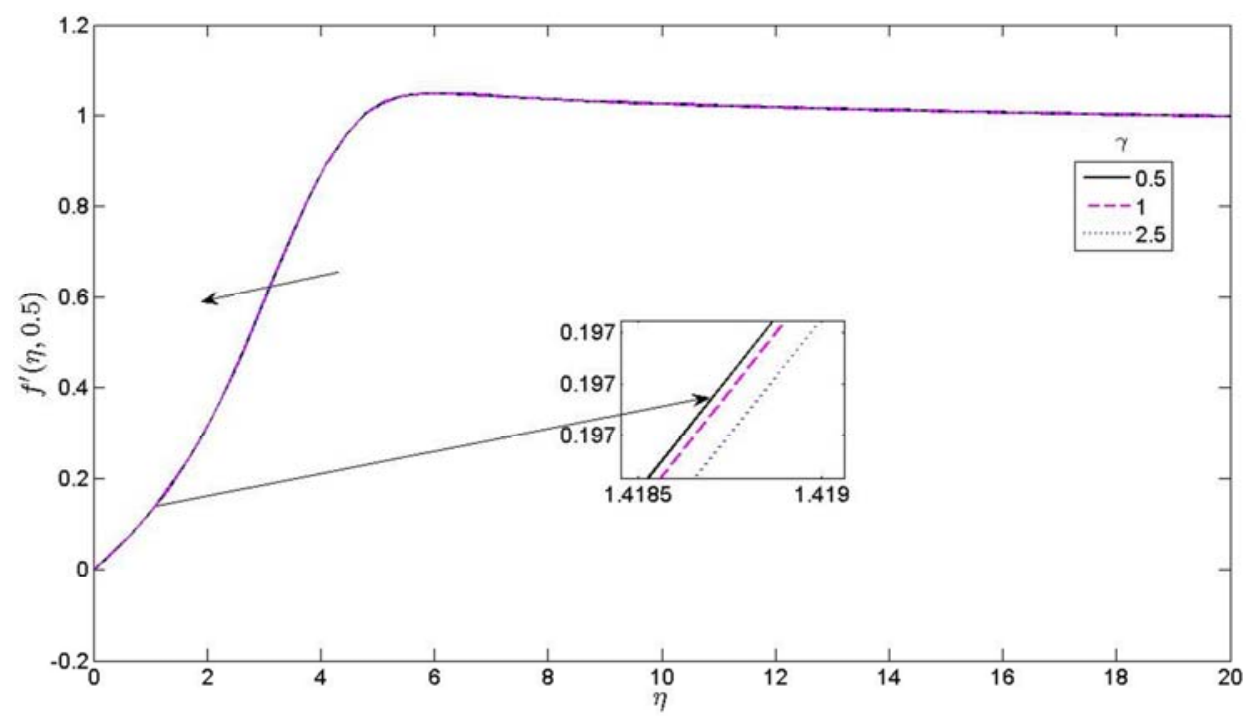

Figure 2. Velocity profiles for different values of chemical reaction $(\gamma)$.

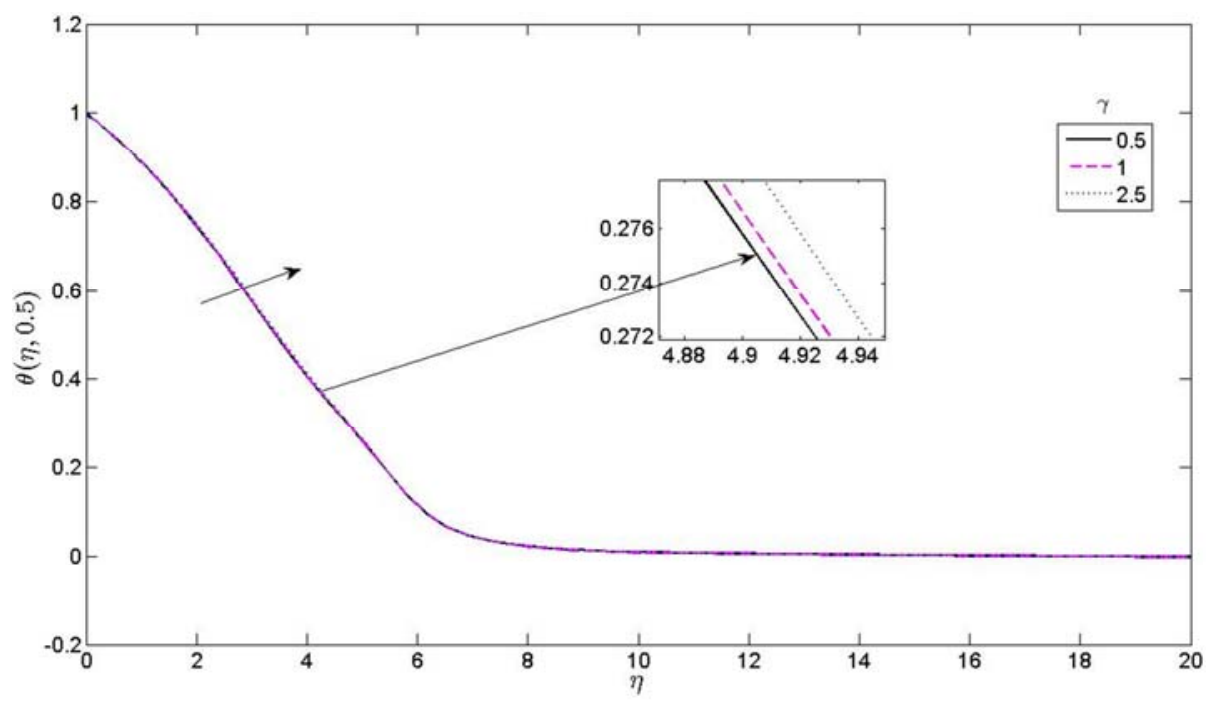

Figure 3. Temperature profiles for different values of chemical reaction $(\gamma)$.

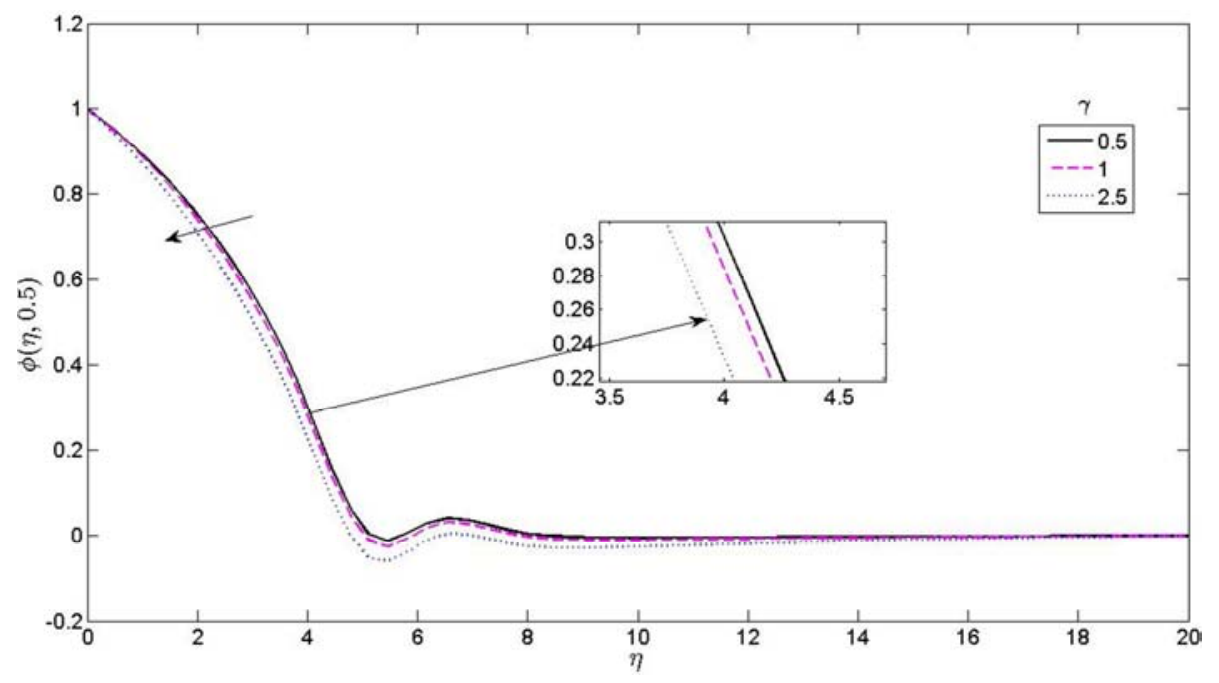

Figure 4. Concentration profiles for different values of chemical reaction $(\gamma)$. 
Figure 5 depicts the velocity distribution $f^{\prime}(\eta, \xi)$ for different values of the buoyancy ratio. Due to the uniform chemical reaction, it is clear that the velocity of the fluid increases with increase of the buoyancy ratio. Figure 6 shows the dimensionless temperature profile for different values of buoyancy ratio. In the presence of uniform chemical reaction, it is seen that the temperature of the fluid decreases with the increase in the buoyancy ratio. Figure 7 portrays the concentration profile of the fluid. It shows concentration of the fluid decrease as the buoyancy ratio increases.

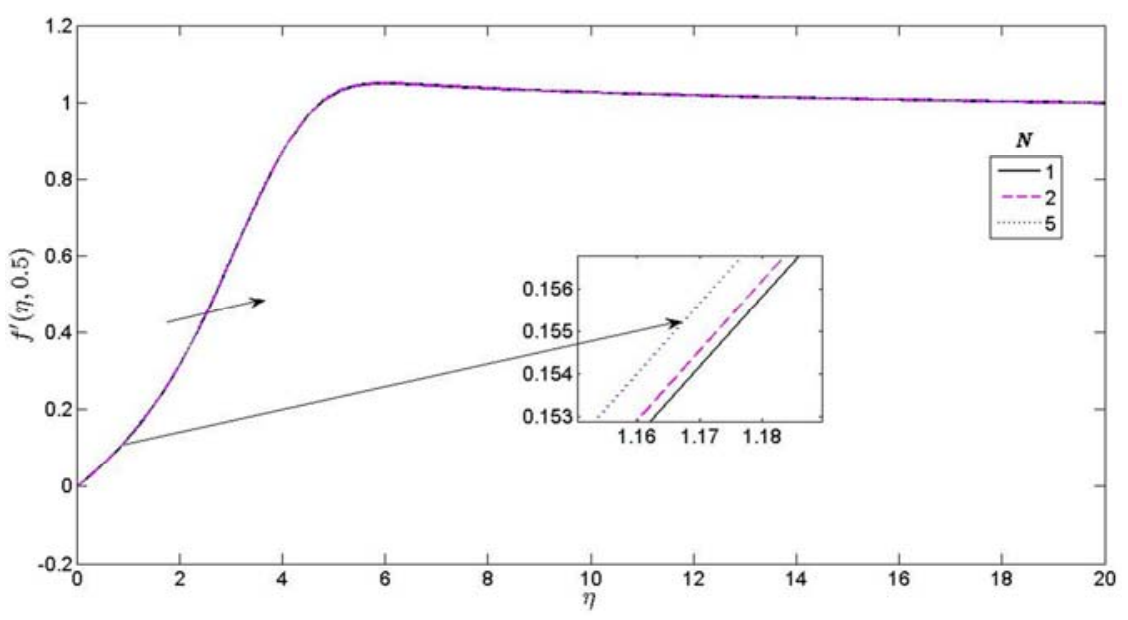

Figure 5. Velocity profiles for different values of buoyancy ratio $(N)$.

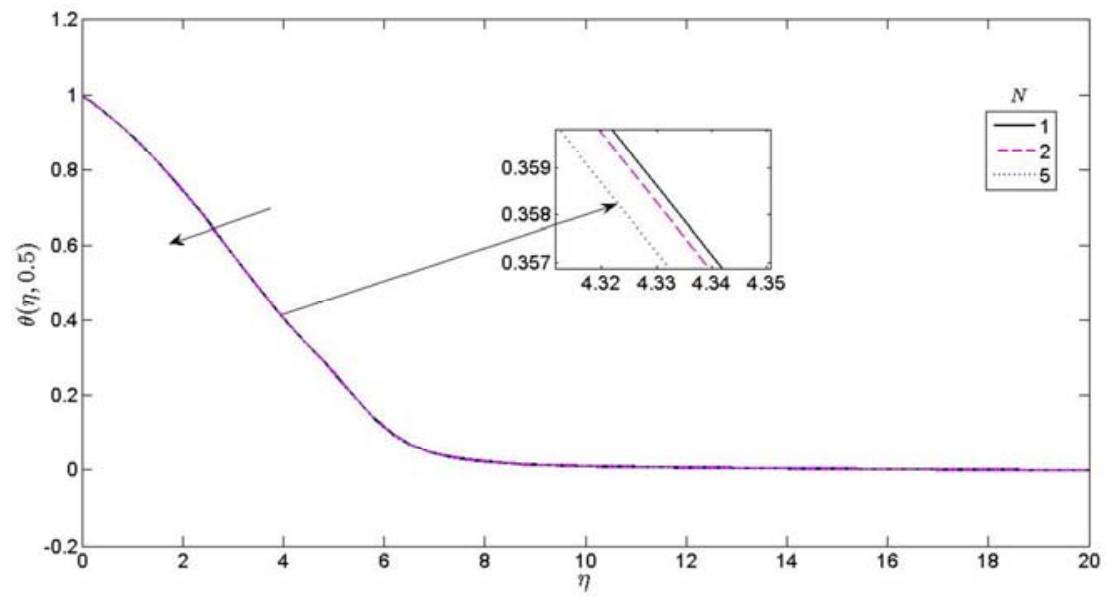

Figure 6. Temperature profiles for different values of buoyancy ratio (N).

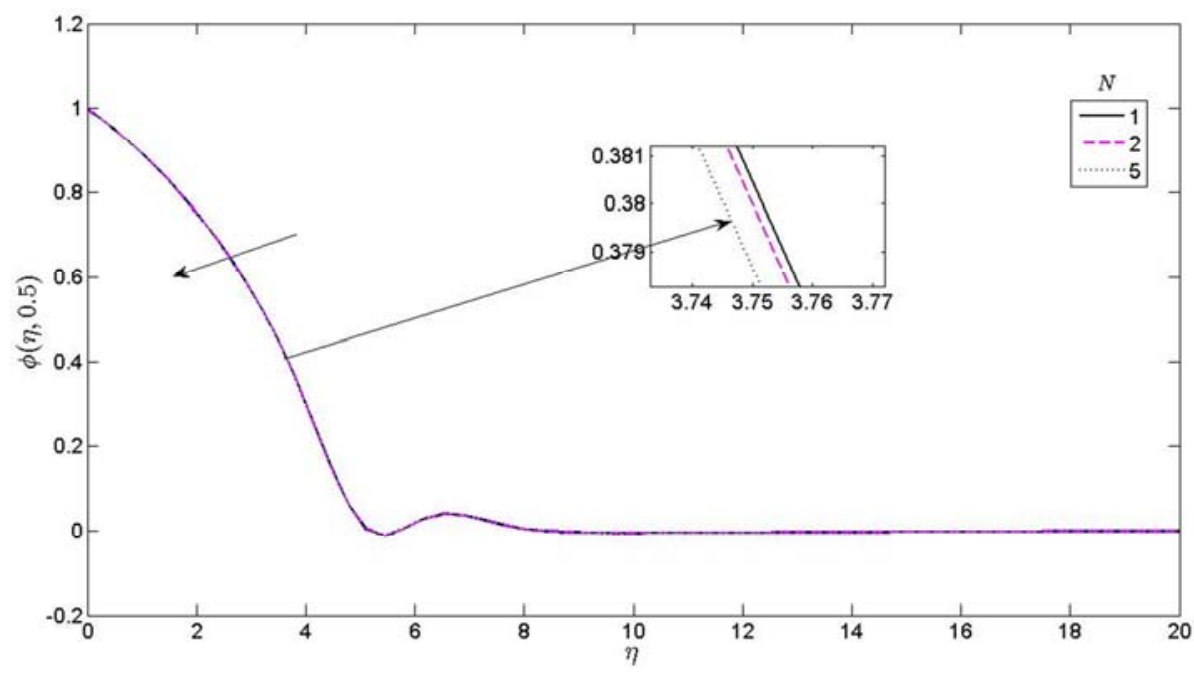

Figure 7. Concentration profiles for different values of buoyancy ratio $(N)$. 
Figure 8 illustrates the dimensionless velocity profiles for different values of heat radiation. In thepresence of uniform chemical reaction and suction, it is clear that the velocity increases in the flow boundary layer thickness with the increase in the heat radiation. Thus, thermal radiation enhances convective flow. Figure 9 shows the temperature profile of the fluid. There was an increase in the temperature profile of the fluid with an increase in the thermal boundary layer thickness as the heat radiation increases. Figure 10 depicts the concentration $\phi^{\prime}(\eta, \xi)$ of the fluid. The concentration increases with the increase in heat radiation.

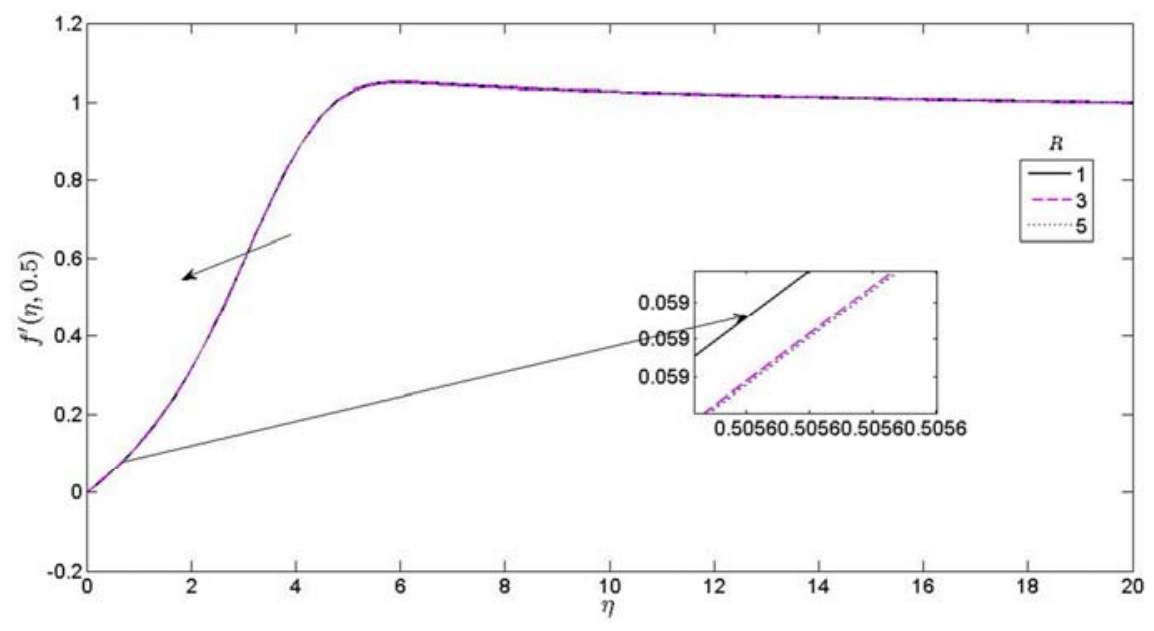

Figure 8. Velocity profiles for different values of radiation parameter (R).

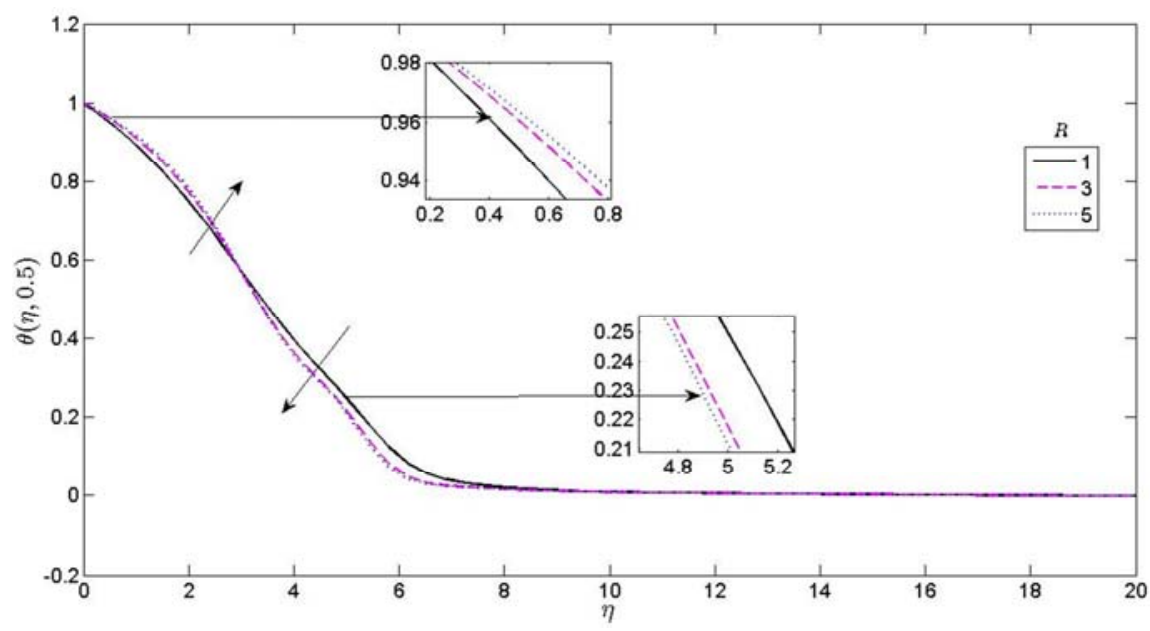

Figure 9. Temperature profiles for different values of radiation parameter $(R)$.

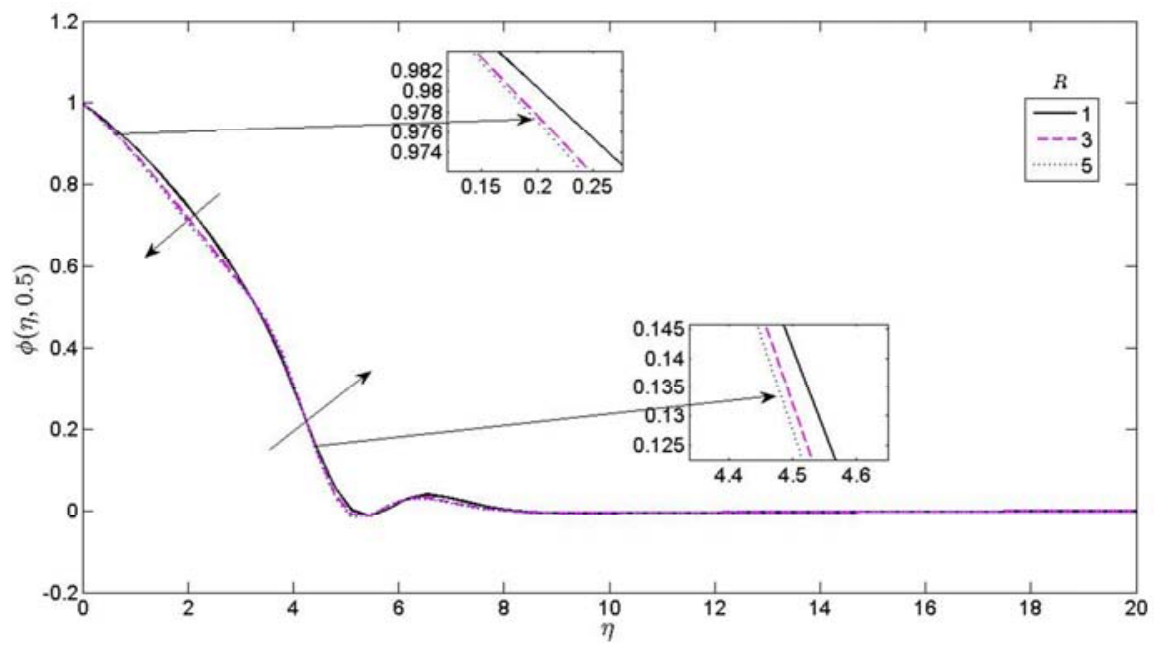

Figure 10. Concentration profiles for different values of radiation parameter ( $R$ ). 
Figure 11 depicts the velocity distribution for different values of soret. There no activity of soret on the velocity of the fluid as the various values of soret increases. Figure 12 reveals the temperature profiles for different values of soret. It shows the decrease in the temperature of the fluid as the soret values increase. Figure 13 exhibits the concentration profiles for different values of soret. There was an increase in the concentration of the fluid as we have various values of soret.

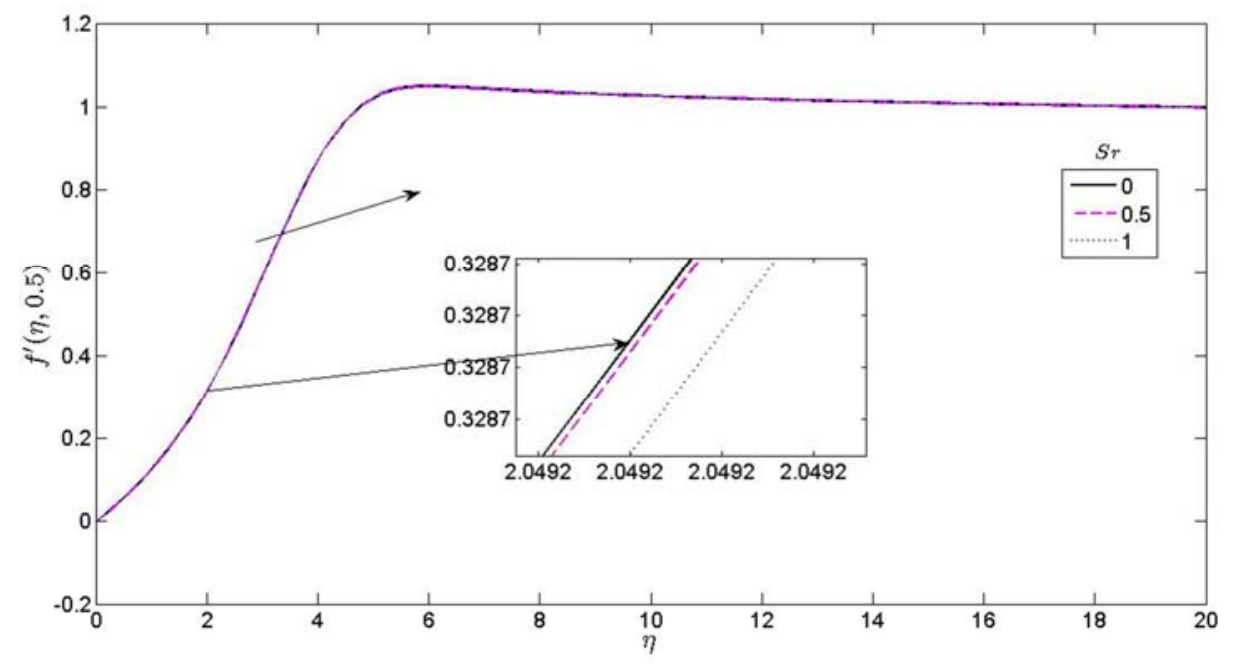

Figure 11. Velocity profiles for different values of soret ( $\mathrm{Sr})$.

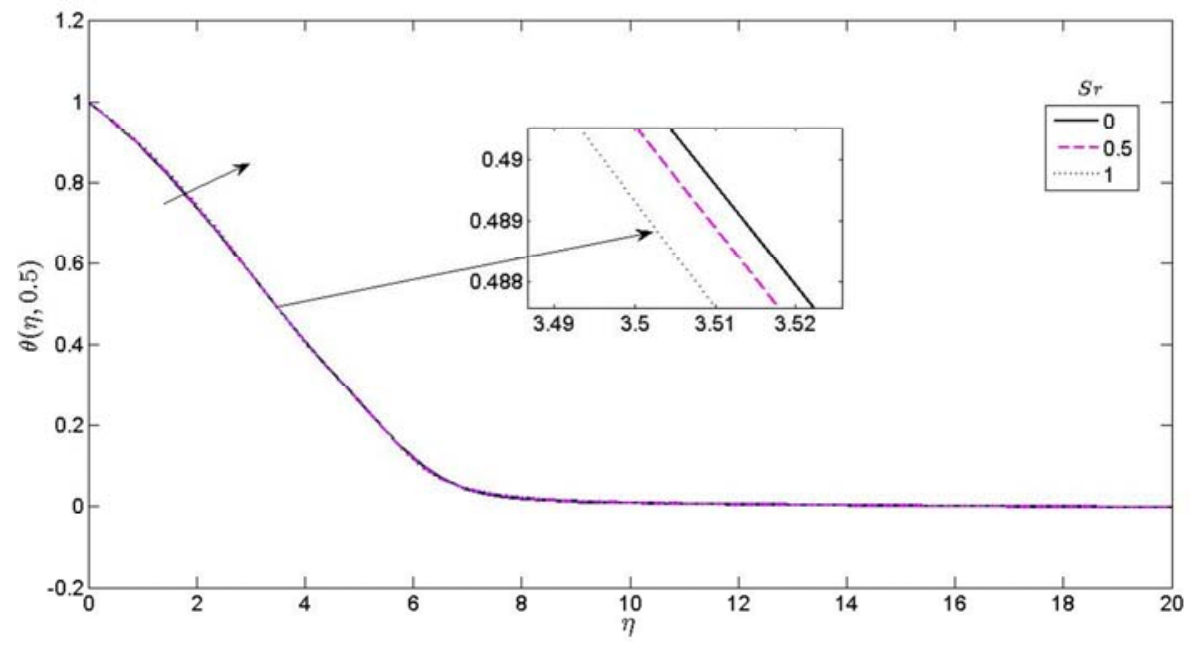

Figure 12. Temperature profiles for different values of soret (Sr).

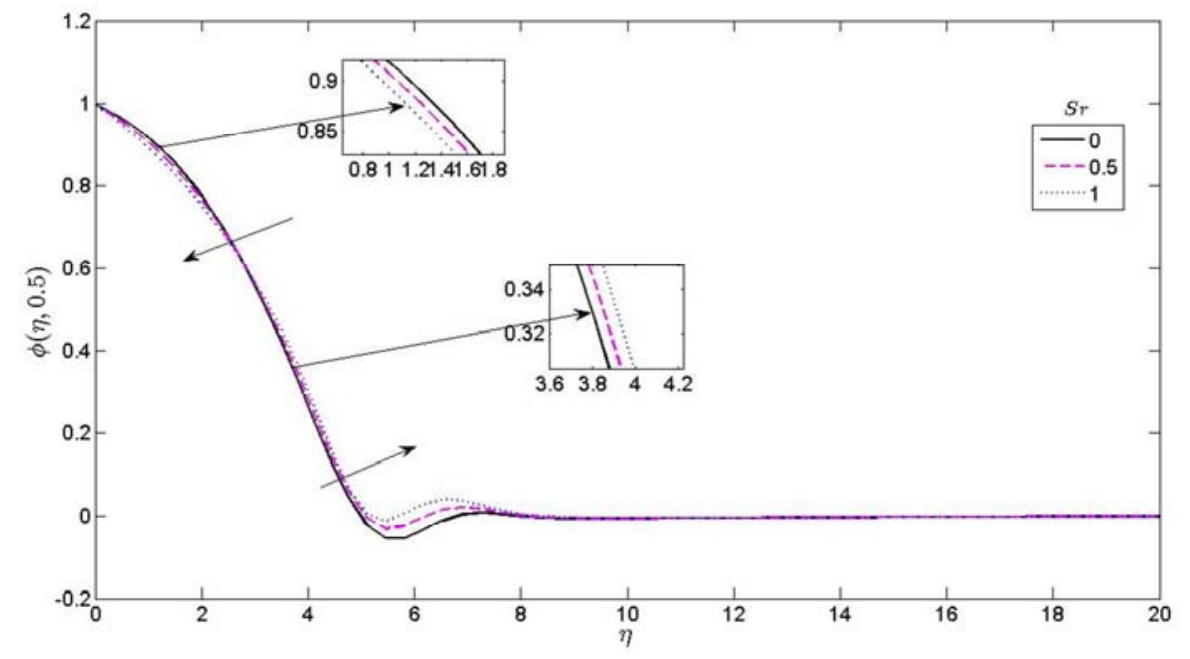

Figure 13. Concentration profiles for different values of soret (Sr). 
Figure 14 portrays the velocity profiles for different values of dufour. There was increase in the values of dufour, but has no effect on the velocity profile of the fluid.

Figure 15 depicts temperature profiles for different values of dufour $(\mathrm{Du})$. The temperature of the fluid increases as the various values of dufour increases. Figure 16 shows the concentration profiles for different values of dufour. With the increase in the values of dufour, there is decrease in the concentration of the fluid.

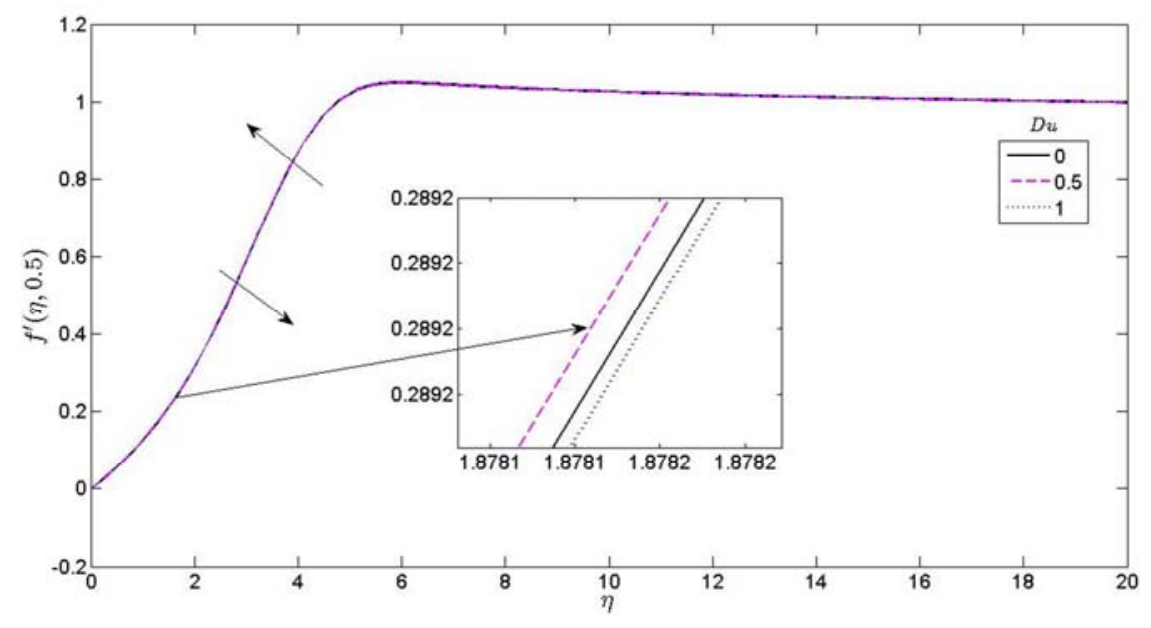

Figure 14. Velocity profiles for different values of dufour (Du).

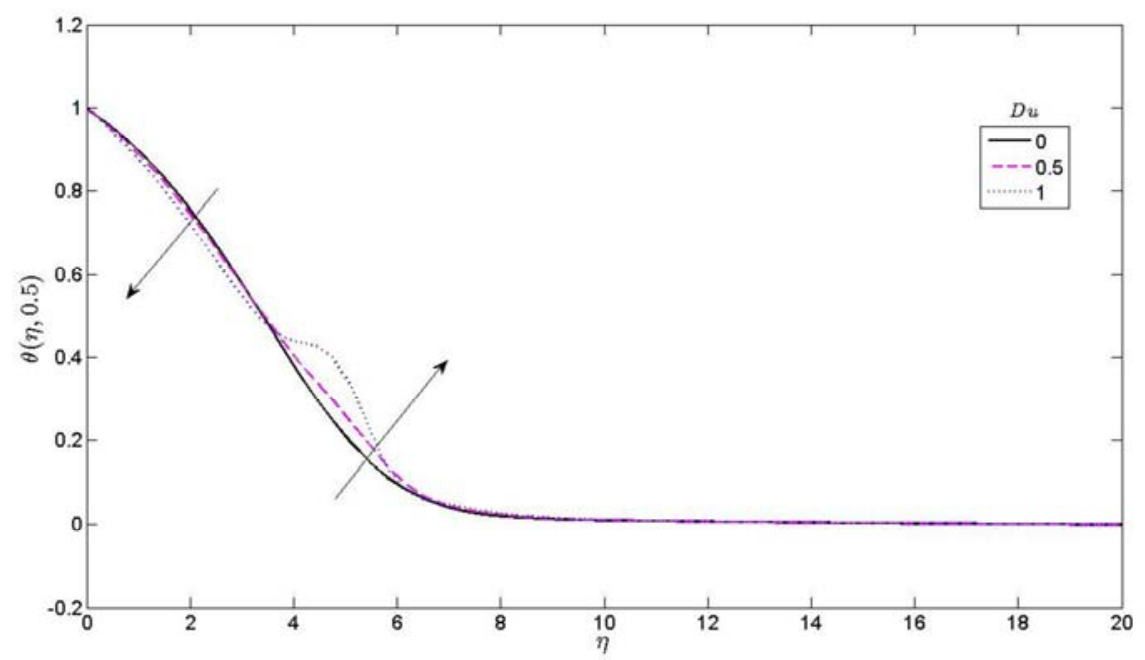

Figure 15. Temperature profiles for different values of dufour (Du).

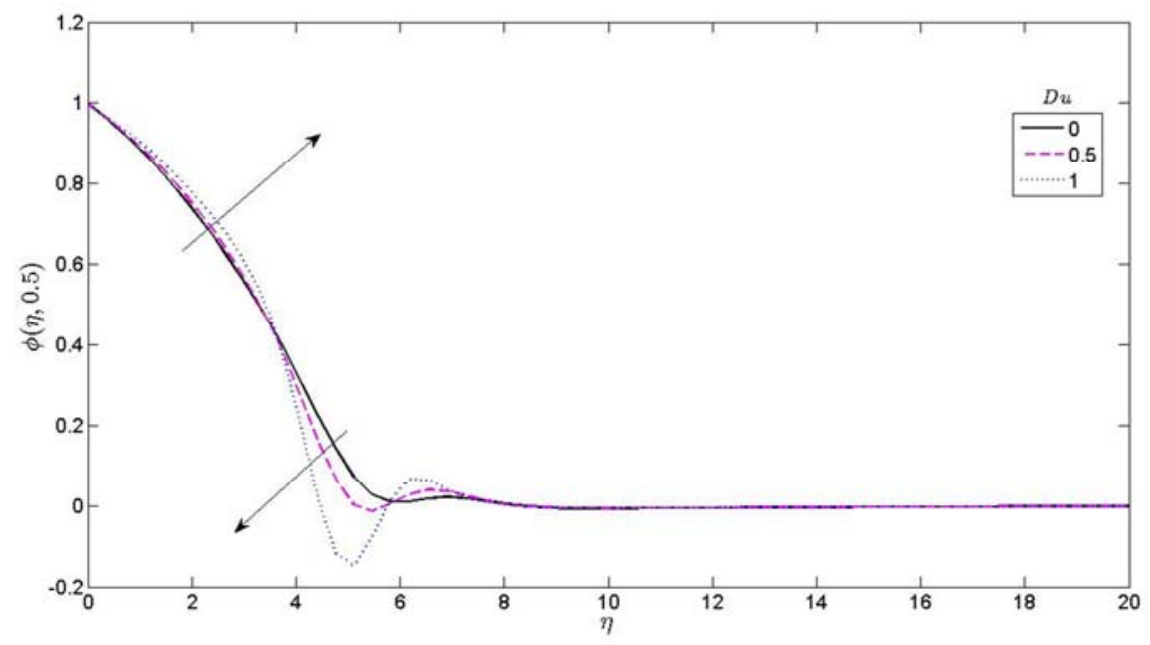

Figure 16. Concentration profiles for different values of dufour (Du). 
Figure 17 reveals the velocity profiles for different values of angle of inclination. The increase in the angle of inclination shows increase in the velocity profile of the fluid. Figure 18 exhibits the temperature profile of the fluid. The increase in the values of the angles of inclination shows no activity in the temperature profile. Figure 19 illustrates the concentration profile of the fluid. The concentration of the fluid has no effect recorded. Representative velocity profiles for three typical angles of inclination ( $\Omega=30^{\circ}, 45^{\circ}$ and $60^{\circ}$ ) are presented. The fact is that as the angle of inclination increases the effect of the buoyancy force due to thermal diffusion decrease by a factor ofsin $\Omega$.

Table 1. Values of local skin friction $f^{\prime \prime}(\xi, 0)$,, local nusselt number $-\theta(\xi, 0)$ and the local sherwood number $-\phi(\xi, 0)$ for $d u$.

\begin{tabular}{lllllllllllll}
\hline $\boldsymbol{S 1}$ & $\boldsymbol{\gamma}$ & $\boldsymbol{P r}$ & $\boldsymbol{N}$ & $\boldsymbol{G} \boldsymbol{r}$ & $\boldsymbol{\delta}$ & $\boldsymbol{S} \boldsymbol{c}$ & $\boldsymbol{\Omega}$ & $\boldsymbol{\xi}$ & $\boldsymbol{\lambda}$ & $\boldsymbol{D u}$ & $\boldsymbol{f}^{\prime \prime}(\boldsymbol{\xi}, \mathbf{0})$ & $-\boldsymbol{\theta}(\boldsymbol{\xi}, \mathbf{0})$ \\
\hline 1 & 0.5 & 0.71 & 2 & 0.5 & 0.5 & 0.62 & $30^{0}$ & 0.5 & 0.5 & 0.0 & 1.4368660 & 0.4752537 \\
1 & 0.5 & 0.71 & 2 & 0.5 & 0.5 & 0.62 & $45^{0}$ & 0.5 & 0.5 & 0.5 & 1.43687020 & 0.3452941 \\
1 & 0.5 & 0.71 & 2 & 0.5 & 0.5 & 0.62 & $60^{0}$ & 0.5 & 0.5 & 1.0 & 1.43687500 & 0.1801338 \\
\hline
\end{tabular}

Table 2. Values of local skin friction $f^{\prime \prime}(\xi, 0)$, local nusselt number $-\theta(\xi, 0)$ and the local sherwood number $-\phi(\xi, 0)$ for sr.

\begin{tabular}{|c|c|c|c|c|c|c|c|c|c|c|c|c|c|}
\hline$S 1$ & $\gamma$ & $\mathrm{Pr}$ & $N$ & $G r$ & $\delta$ & $S c$ & $\Omega$ & $\xi$ & $\lambda$ & $S r$ & $f^{\prime \prime}(\xi, 0)$ & $-\boldsymbol{\theta}(\xi, \mathbf{0})$ & $-\phi(\xi, 0)$ \\
\hline 1 & 0.5 & 0.71 & 2 & 0.5 & 0.5 & 0.62 & $30^{\circ}$ & 0.5 & 0.5 & 0.0 & 1.4368586 & 0.3632003 & 1.1081819 \\
\hline 1 & 0.5 & 0.71 & 2 & 0.5 & 0.5 & 0.62 & $45^{0}$ & 0.5 & 0.5 & 0.5 & 1.4368693 & 0.37373015 & 1.0256579 \\
\hline 1 & 0.5 & 0.71 & 2 & 0.5 & 0.5 & 0.62 & $60^{\circ}$ & 0.5 & 0.5 & 1.0 & 1.4368773 & 0.38495278 & 0.9391546 \\
\hline
\end{tabular}

Table 3. Values of local skin friction $f^{\prime \prime}(\xi, 0)$, local nusselt number $-\theta(\xi, 0)$ and the local sherwood number $-\phi(\xi, 0)$ for $r$.

\begin{tabular}{llllllllllllll}
\hline $\boldsymbol{S 1}$ & $\boldsymbol{\gamma}$ & $\boldsymbol{P r}$ & $\boldsymbol{N}$ & $\boldsymbol{G} \boldsymbol{r}$ & $\boldsymbol{\delta}$ & $\boldsymbol{S c}$ & $\boldsymbol{\Omega}$ & $\boldsymbol{\xi}$ & $\boldsymbol{\lambda}$ & $\boldsymbol{R}$ & $\boldsymbol{f}^{\prime \prime}(\boldsymbol{\xi}, \mathbf{0})$ & $-\boldsymbol{\theta}(\boldsymbol{\xi}, \mathbf{0})$ & $-\boldsymbol{\phi}(\xi, \mathbf{0})$ \\
\hline 1 & 0.5 & 0.71 & 2 & 0.5 & 0.5 & 0.62 & $30^{0}$ & 0.5 & 0.5 & 0.0 & 1.4369958 & 0.1000000 & 1.1082348 \\
1 & 0.5 & 0.71 & 2 & 0.5 & 0.5 & 0.62 & $45^{0}$ & 0.5 & 0.5 & 0.5 & 1.4368693 & 0.3737301 & 1.0256579 \\
1 & 0.5 & 0.71 & 2 & 0.5 & 0.5 & 0.62 & $60^{0}$ & 0.5 & 0.5 & 1.0 & 1.4368472 & 0.4747728 & 0.9997569 \\
\hline
\end{tabular}

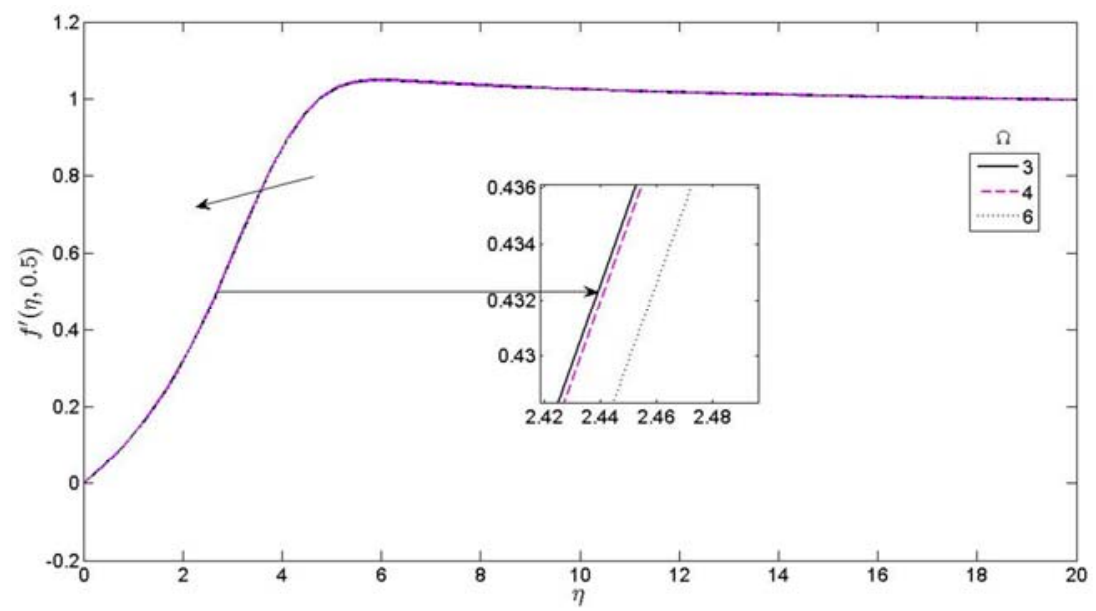

Figure 17. Velocity profiles for different values of angle of inclination $(\Omega)$.

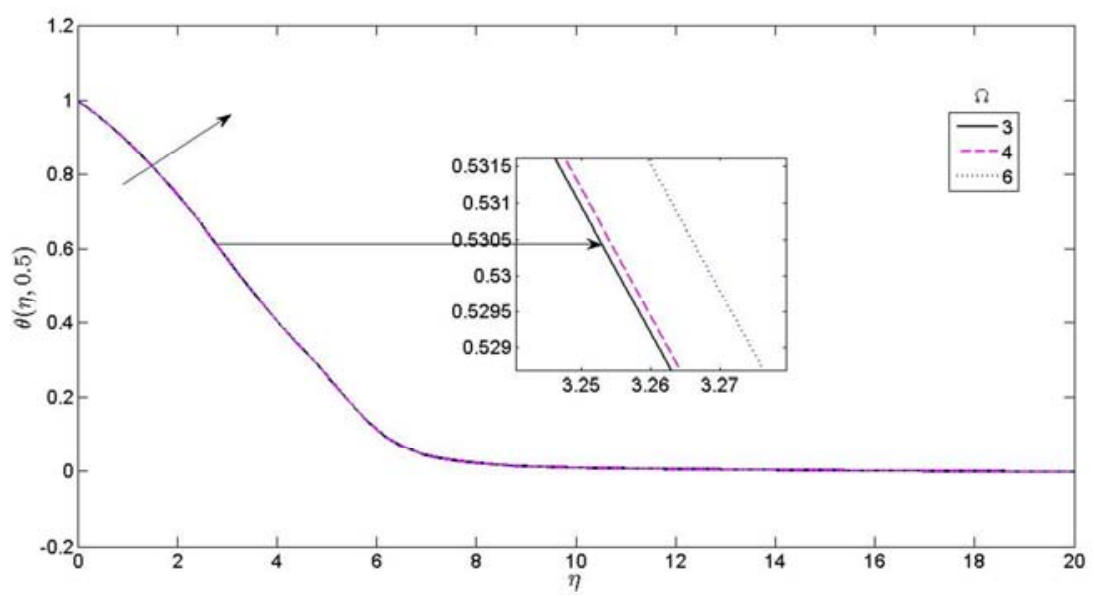

Figure 18. Temperature profiles for different values of angle of inclination $(\Omega)$. 


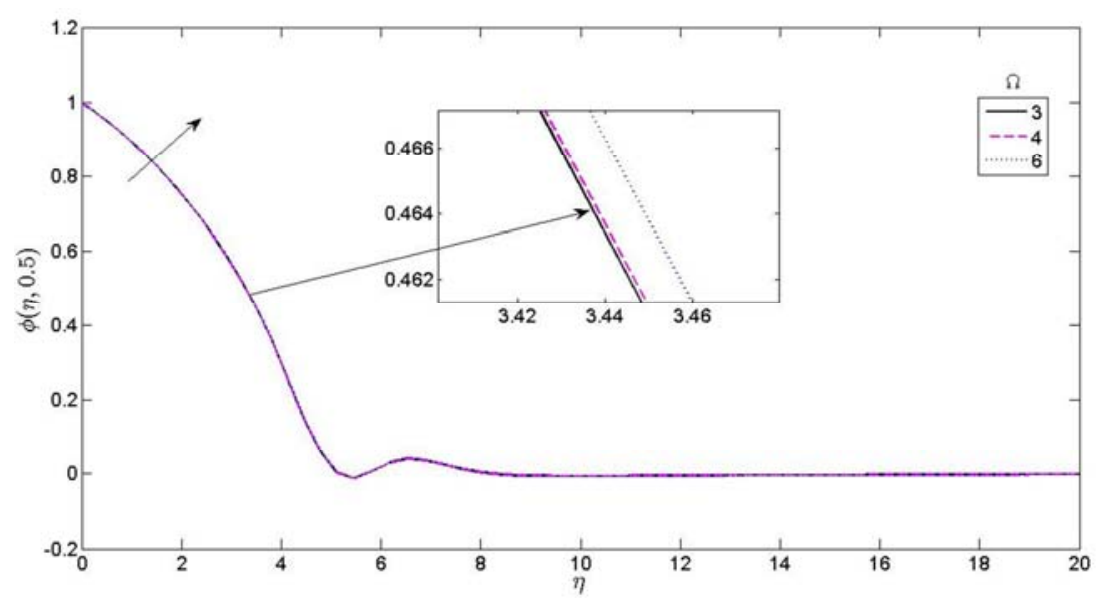

Figure 19. Concentration profiles for different values of angle of inclination $(\Omega)$.

The effects of soret number, dufour number and radiation parameter on the local skin-friction coefficient, the local nusselt number and the local sherwood number as shown in Tables 1, 2 and 3 above. The increase in soret number shows that the local skin friction and the local nusselt number increase while local sherwood number decrease. The increase of dufour number indicates an increase in skin friction and local sherwood number and decrease in local nusselt number. The increase of radiation parameter shows the decrease in local skin friction and local sherwood number while local nusselt number increases.

\section{Conclusion}

The present study gives the numerical solution of boundary layer flow over porous wedge in the presence of heat radiation, soret and dufour effects. There are many parameters involved in the final form of the mathematical model.

It shows the effects of the controlling parameters on the flow's velocity, temperature and concentration profiles in the boundary layer. We conclude that:

1. a decrease in velocity and temperature of the fluid with the increase in the heat radiation. The concentration profile increases with the increase in heat radiation.

2. the decrease in the temperature of the fluid as the soret values increase. There was an increase in the concentration of the fluid as we have various values of soret.

3. the temperature of the fluid increases as the various values of dufour increases, while there is decrease in the concentration of the fluid.

4. decrease in the temperature and concentration of the fluid as the suction velocity increases. Hence suction reduces the growth of the thermal and concentration boundary layers.

5. the velocity of the fluid increases with increase of the buoyancy ratio. The temperature and concentration of the fluid decrease with the increase in the buoyancy ratio.

The presented analysis has also shown that the flow field is appreciably influenced by the dufour and soret effects. Therefore, we can conclude that the dufour and soret effects on boundary layer of fluids as it is expected that this research may prove to be useful for the study of movement of fluid (oil, gas and water) through the reservoir of an oil or gas field, in the movement of underground water and also the filtration and purification of water processes.

\section{Nomenclature}

$U_{0}(x)$-velocity of the flow

$u, v-$ velocity components in $x$ and $y$ respectively

$x, y-$ coordinate system

$g-$ gravitational force due to acceleration

$D_{m}-$ coefficient of mass diffusivity

$C_{p}-$ specific heat at constant pressure

$K_{r}$ - chemical reaction parameter

$N-$ buoyancy ratio

$\mathrm{Pr}-$ prandtl number

$S c-$ schmidt number

$\mathrm{Gr}$ - grashof number

$S 1$ - suction or injection parameter

$C_{\infty}-$ free stream concentration

$C-$ fluid concentration

$T$ - fluid temperature

$C_{w}-$ concentration at the wall

$T_{w}$ - temperature at the wall

$T_{m}$ - mean fluid temperature

$T_{\infty}$ - free stream temperature

$C-$ concentration at any point in the flow field

$C_{s}-$ concentration susceptibility

$D u$ - dufour number

$\mathrm{Sr}$ - soret number

$S c-$ schmidt number

$R$ - radiation parameter

$f$ - dimensionless stream function

$f^{\prime}-$ dimensionless velocity function

$K_{T}$ - thermal diffusion ratio

$\mathrm{Nu}$ - nusselt number

$C_{f}-$ local friction number

$S h$ - sherwood number 
$p-$ pressure

$m$ - falkner - skan power - law parameter

$q_{r}-$ radiative heat flux

$Q_{0}$-amount of heat generated or absorbed per unit volume

\section{Greek Symbols}

$\propto-$ fluid thermal diffusivity

$\beta$ - viscosity

$\beta_{T}$-thermal expansion coefficient

$\beta_{C}$-concentration expansion coefficient

$\beta_{1}$-hartree pressure

$\beta_{2}$ - constant

$\delta-$ internal heat generation or absorption coefficient

$\xi-$ dimensionless distance along the wedge

$\psi$-dimensionless stream function

$\eta$-dimensionless similarity variable

$\Omega$-angle of inclination

$\rho$-density of the fluid

$\sigma$-stefan-boltzmann constant

$\mu-$ mean absorption coefficient

$\theta$-dimensionless temperature

$\phi$-dimensionless concentration

$\vartheta$-kinematic viscosity

$\lambda$-porous medium parameter

$\gamma$-chemical reaction parameter

\section{References}

[1] M. H. Shojaefard, A. R. Noorpoor, A. Avanesians, M. Ghaffapour, (2005). Numerical investigation of flow control by suction and injection on a subsonic airfoil, AM. J. Appl. Sci., 20, 1474- 1480 .

[2] A. I Braslow, (1999). A history of suction type laminar flow control with emphasis on flight research, American Institute of Aeronautics and Astronautics, Washington, D. C., 15.

[3] SudhagarPalani, Peri K. Kameswaran and B. Rushi Kumar, (2018), Non-darcy effects on mixed convective nanofluid over a wedge in a porous medium, Journal of Porous Media, 21(9):781-791.

[4] M. B. K. Moorthy, T. Kannan, K. Senthilvadivu, (2013). Soret and Dufour Effects on Natural Convection Heat and Mass Transfer Flow past a Horizontal Surface in a Porous Medium with Variable Viscosity, Wseas Transactions On Heat And Mass Transfer, 121-130.

[5] S. Shivaiah and J AnandRao, (2011). Effects Of Soret, Dufour And Thermal Radiation On Unsteady Mhd Free Convection Flow Past An Infinite Vertical Porous Plate In The Presence Of Chemical Reaction, Int. J. Of Appl. Math and Mech. 7 (13): 58-76.

[6] A. J. Omowaye, A. I. Fagbade, A. J. Ajayi, (2015). Dufour and soret effects on steady MHD convective flow of a fluid ina porous medium with temperature dependent viscosity: Homotopy analysis approach, Journal of the NigerianMathematical Society, http://dx.doi.org/10.1016/j.jnnms.2015.08.001.

[7] M. M. Rashidi, M. Ali, N. Freidoonimehr, B. Rostami, and M. Anwar Hossain, (2014). Mixed Convective Heat Transfer for MHD Viscoelastic Fluid Flow over a Porous Wedge with Thermal Radiation, Advances in Mechanical Engineering, Volume, Article ID 735939, 10 pages, (http://dx.doi.org/10.1155/2014/735939).

[8] I. Muhaimin, R. Kandasamy, Azme B. Khamis, Rozani bin Roslan, (2013). Impact of thermophoresis particle deposition and chemical reaction on unsteady non-Darcy mixed convective flow over a porous wedge in the presence of temperature-dependent viscosity, Meccanica, 48: 1415-1430, (DO1 10.10071 11012-012-9675-6).

[9] B. Lavanya and A. LeelaRatnam, (2014). Dufour and soret effects on steady MHD free convective flow past a vertical porous plate embedded in a porous medium with chemical reaction, radiation heat generation and viscous dissipation, Advances in Applied Science Research, 5(1):127-142.

[10] R. Kandasamy, B. A. Wahid, M. Raj, A. B. Khamis, (2006). Effects of chemical reaction, heat and mass transfer on boundary layer flow over a porous wedge with heat radiation in the presence of suction or injection, Theoret. Appl. Mech., $33,123-148$.

[11] Wubshet Ibrahim and Ayele Tulu, (2019). Magnetohydrodynamic (MHD) boundary layer flow past a wedge with heat transfer and viscous effects of nanofluid embedded in porous media, Mathematical Problems in Engineering, Volume 2019, Article ID 4507852, 12 pages. https://doi.org/10.1155/2019/4507852

[12] S. S. Motsa, P. G. Dlamini, M. Khumalo, (2014). Spectral Relaxation Method and Spectral Quasilinearalisation Method for Solving Unsteady Boundary Layer Flow Problems, Advances in Mathematical Physics, Vol. 2014, Article ID 341964, 12 pages, doi:10.1155/2014/341964.

[13] J. Boussinesq, (1897). Theory of turbulent and tumultuous flow of liquids in prismatic channels of large cross- sections (pipes and open channels) when the flow is uniform, GauthierVillars Paris Vol. (1), Open Library OL7070543M.

[14] N. G. Kafoussias, N. D., Nanousis, (1997). Magnetohydrodynamic laminar boundary layer flow over a wedge with suction or injection. Can, J. Physics, 75, 733.

[15] S. S Motsa, V. M. Magagula and P. Sibanda, (2014). A Bivariate Chebyshev Spectral Collocation Quasilinearization Method for Non-linear Evolution Parabolic Equations, The Scientific World Journal, vol. 2014, Article ID 581987, 13 pages, doi:10.1155/2014/581987.

[16] C. Canuto., M. Y Hussaini, A. Quarteroni, and T. A. Zang, (1988). Spectral Methods in Fluid Dynamics, Springer-Verlag, Berlin, http://dx.doi.org/10.1007/978-3-642-84108-8.

[17] L. N. Trefethen, (2000). Spectral Methods in MATLAB. SIAM, Philadelphia, http://dx.doi.org/10.1137/1.9780898719598. 